This item was submitted to Loughborough's Research Repository by the author.

Items in Figshare are protected by copyright, with all rights reserved, unless otherwise indicated.

\title{
Information and energy cooperation in cognitive radio networks
}

\section{PLEASE CITE THE PUBLISHED VERSION}

https://doi.org/10.1109/TSP.2014.2310433

PUBLISHER

(c) IEEE

VERSION

AM (Accepted Manuscript)

LICENCE

CC BY-NC-ND 4.0

REPOSITORY RECORD

Zheng, Gan, Zuleita Ho, Eduard A. Jorswieck, and Bjorn Ottersten. 2014. "Information and Energy Cooperation in Cognitive Radio Networks". Loughborough University. https://hdl.handle.net/2134/34760. 


\title{
Information and Energy Cooperation in Cognitive Radio Networks
}

\author{
Gan Zheng, Senior Member, IEEE, Zuleita Ho, Member, IEEE, Eduard A. Jorswieck, Senior Member, IEEE, and \\ Björn Ottersten, Fellow, IEEE
}

\begin{abstract}
Cooperation between the primary and secondary systems can improve the spectrum efficiency in cognitive radio networks. The key idea is that the secondary system helps to boost the primary system's performance by relaying and in return the primary system provides more opportunities for the secondary system to access the spectrum. In contrast to most of existing works that only consider information cooperation, this paper studies joint information and energy cooperation between the two systems, i.e., the primary transmitter sends information for relaying and feeds the secondary system with energy as well. This is particularly useful when the secondary transmitter has good channel quality to the primary receiver but is energy constrained. We propose and study three schemes that enable this cooperation. Firstly, we assume there exists an ideal backhaul between the two systems for information and energy transfer. We then consider two wireless information and energy transfer schemes from the primary transmitter to the secondary transmitter using power splitting and time splitting energy harvesting techniques, respectively. For each scheme, the optimal and zero-forcing solutions are derived. Simulation results demonstrate promising performance gain for both systems due to the additional energy cooperation. It is also revealed that the power splitting scheme can achieve larger rate region than the time splitting scheme when the efficiency of the energy transfer is sufficiently large.
\end{abstract}

Index Terms-Cognitive radio, cognitive relaying, information and energy cooperation, energy harvesting, wireless energy transfer.

\section{INTRODUCTION}

\section{A. Motivation}

Cooperative cognitive radio networks (CCRN) have been a new paradigm to improve the spectrum efficiency of a cognitive radio (CR) system where the primary and secondary systems actively seek opportunities to cooperate with each other.

Gan Zheng is with School of Computer Science and Electronic Engineering, University of Essex, UK, E-mail: ganzheng@essex.ac.uk. He is also affiliated with Interdisciplinary Centre for Security, Reliability and Trust (SnT), University of Luxembourg, Luxembourg.

Zuleita Ho was with Communications Laboratory, Dresden University of Technology, Dresden, Germany. She is now a senior engineer in the Advanced Communications Laboratory, DMC R\&D Center, Samsung Electronics, Korea, E-mail:zuleita.ho@samsung.com.

Eduard A. Jorswieck is with Communications Laboratory, Dresden University of Technology, Dresden, Germany, E-mail:eduard.jorswieck@tudresden.de. This work has been performed in the framework of the European research projects DIWINE and ACROPOLIS, which are partly funded by the European Union under its FP7 ICT Objective 1.1 - The Network of the Future.

Björn Ottersten is with the Interdisciplinary Centre for Security, Reliability and Trust (SnT), University of Luxembourg, Luxembourg, E-mail: bjorn.ottersten@uni.lu.

Copyright (c) 2013 IEEE. Personal use of this material is permitted. However, permission to use this material for any other purposes must be obtained from the IEEE by sending a request to pubs-permissions@ieee.org.
CCRN have many advantages over existing non-cooperative CR schemes. It is a win-win strategy for both systems in the sense that the secondary transmitter (ST) helps relay the traffic from the primary transmitter (PT) to the primary user (PU), and in return can utilize the primary spectrum to serve its own secondary user (SU). This is especially preferred by the primary system when the PU's quality-of-service (QoS) cannot be met by the primary system itself. Compared to the conventional interweave CR technique [1] which is an opportunistic access scheme, the cooperation scheme does not require the ST to wait and sense the spectrum holes for transmission; unlike the underlay technique [2] which sets limit on the interference to the primary system, the cooperation scheme focuses on the end performance, e.g., the PU rate or the signal to interference plus noise ratio (SINR), thus the ST is no longer restricted to transmit with low power.

However, most existing CCRN assume that the cooperation is only at the information level. One problem is that even when the ST has good channel quality to help serve the PU but is energy constrained, the cooperation is still not possible. This is a commonly seen situation when the ST is a lowpower relay node rather than a powerful base station (BS). This motivates us to propose the cooperation between the primary and secondary systems at both information and energy levels, i.e., the PT will transmit both information and energy to the ST, in exchange for the ST to relay the primary information. Compared to the existing CCRN with only information cooperation, this scheme creates even stronger incentives for both systems to cooperate and substantially improves the system overall spectrum efficiency. It can be seen as an enhanced win-win strategy. The energy cooperation can be enabled by the recently proposed energy harvesting or wireless energy transfer techniques [25]. In the sequel, we will briefly review the literature about CCRN and energy cooperation.

\section{B. Related Works}

1) CCRN: Early works about CCRN are mainly from the viewpoint of information theory [3]-[6] assuming non-causal primary message available at the ST, where the ST employs dirty paper coding (DPC) to remove interference from the SU due to the primary signal. Using multiple antennas and noncausal primary message at the ST, the optimal beamforming is studied for both cases using DPC [7] and linear precoding [8]. However, these schemes require non-causal primary information at the ST; therefore they are hard to implement in practice and only provide an outer bound on the achievable primary-secondary rate region. 
As to more practical CCRN, three-phase cooperation protocols between primary and cognitive systems are proposed to exploit primary resources in time and frequency domain [10], [11]. The ST uses the first two phases to listen and forward the primary traffic; in return, the last phase is exclusively reserved for the ST to transmit its own signal to the SU. The use of multiple antennas and beamforming at the ST provides additional degree of freedom for the concurrent primary-cognitive cooperation. The zero-forcing (ZF) beamforming technique and the optimal beamforming solution have been studied in [12][13] and [14], respectively. Different from the singleantenna case, the ST with multiple antennas requires only two phases: Phase I is the same as that in the single-antenna case while in Phase II, the ST can both relay the primary signal and transmit its own signal due to its ability of signal separation in the spatial domain. Recently full-duplex radio has been investigated in [16] for CCRN which requires only one phase and it can efficiently enlarge the achievable rate region. Both the uncoordinated underlay cognitive radio scenario and the coordinated overlay cognitive radio scenario that consists of a message-learning phase followed by a communication phase are studied in [9].

2) Energy Cooperation: Energy cooperation is a promising solution to prolong the network lifetime despite the possible loss during the process of energy transfer. In case of power line systems, joint communication and energy cooperation is investigated in [19] for the coordinated multi-point downlink cellular networks. The base stations (BSs) powered by renewable energy are connected by a power line to enable simultaneous data and energy sharing. The proposed joint communication and energy cooperation solution are shown to substantially improve the downlink throughput for energy harvesting $(\mathrm{EH})$ systems, as compared to the case without energy cooperation. In a similar scenario, the optimal energy cooperation algorithms are designed in [20] for both cases where the renewable energy profile and energy demand profile are deterministic and stochastic.

As to wireless energy transfer, recently the radio frequency (RF) EH technology has emerged as a new solution where the electromagnetic radiation in the environment is captured by the receiver antennas and converted into useful energy. Thanks to recent advances in antenna and rectenna circuit design, there has been great progress towards improving the efficiency of wireless energy transfer, for instance, Powerharvester receivers provided by Powercast can achieve conversion efficiency as high as $70 \%$ in some scenarios [21]. A sensor node powered by a cellular Base Transceiver Station (BTS) at a distance of $200 \mathrm{~m}$ from the BTS was implemented in [22]. RF-EH technique also enables simultaneous transfer of information and energy using RF signals [23][24]. Two practical receiver structures to decode information and EH called "time switching" and "power splitting", are proposed in [25]. "Power splitting" divides the received signal into two parts, one for harvesting energy and the other for information decoding. "Time switching" uses dedicated time slots for harvesting energy and the rest for data transmission. Dynamic switching between information decoding and RF EH is proposed in [26] then further studied in [27] for a cooperative relaying scenario with a discrete-level battery at the RF-EH relay node.

When the wireless terminals have RF EH capabilities, energy cooperation provides more performance gain in additional to the usual information cooperation in cooperative communications. Energy cooperation is considered in [28] for several basic multi-user network structures including relay channel, two-way channel and multiple access channel. The optimal energy management policies that maximize the system throughput within a given duration is studied. A more relevant one to our work is [29], where an energy constrained relay node harvests energy from the received RF signal and uses that harvested energy to forward the source information to the destination, therefore the relay does not need external power supply. Both time switching and power switching relaying protocols are proposed to enable $\mathrm{EH}$ and information processing at the relay. Outage capacity and ergodic capacity are also derived.

\section{Contribution}

In this paper, we propose two-level cooperation between the primary and the secondary systems to achieve better use of the spectrum. The first one is information cooperation, where the PT broadcasts the primary signal and after receiving it, the ST retransmits it to the PU; the second one is energy cooperation, where the PT transmits power to the ST via either cable or wireless medium, such that the ST can obtain extra power to help the PT, as well as serve its own SU. The ST may have good channel condition to the PU, but lacks spectrum and energy, therefore, the two-level cooperation substantially increases the chance that the ST can assist the primary transmission and use the primary spectrum. We assume the ST is equipped with multiple antennas, and deals with the beamforming design to characterize the achievable primarysecondary rate region. In particular, we study the problem of maximizing the SU rate subject to the PU rate and ST power (including harvested power) constraints by optimizing the beamforming design at the ST for $\mathrm{EH}$ and relay processing.

We propose three schemes that enable information as well as energy cooperation: i) ideal cooperation where we assume the primary information is non-causally known at the ST and the transmit power can be shared between the PT and the ST; ii) power splitting scheme where the ST uses part of received signal for information decoding and the rest for energy harvesting; and iii) time splitting scheme where a fraction of time is reserved for wireless energy transfer from the PT to the ST and the rest of time is used for information listening and forwarding. For each scheme, we propose efficient algorithms to optimally solve the above mentioned optimization problem. In addition, we derive low-complexity solutions based on $\mathrm{ZF}$ criterion, which provide some insights on the impacts of system parameters.

\section{Notations}

Throughout this paper, the following notations will be adopted. Vectors and matrices are represented by boldface lowercase and uppercase letters, respectively. $\|\cdot\|$ denotes the 
Frobenius norm. $(\cdot)^{\dagger}$ denotes the Hermitian operation of a vector or matrix. $\mathbf{A} \succeq \mathbf{0}$ means that $\mathbf{A}$ is positive semi-definite. I denotes an identity matrix of appropriate dimension. $E[\cdot]$ denotes the expectation. $\mathbf{x} \sim \mathcal{C N}(\mathbf{m}, \mathbf{\Theta})$ denotes a vector $\mathbf{x}$ of complex Gaussian elements with a mean vector of $\mathbf{m}$ and a covariance matrix of $\Theta$. $\Pi_{\mathbf{X}}$ denotes the orthogonal projection onto the column space of $\mathbf{X}$ while $\Pi_{\mathbf{X}} \frac{\perp}{\text { denotes the orthogonal }}$ projection onto the orthogonal complement of the column space of $\mathbf{X}$. We further define $[x]_{0}^{1} \triangleq \min (1, \max (0, x))$. $\operatorname{diag}(\mathbf{v})$ denotes a diagonal matrix with diagonal elements as the elements of $\mathbf{v}$.

\section{General System Setting}

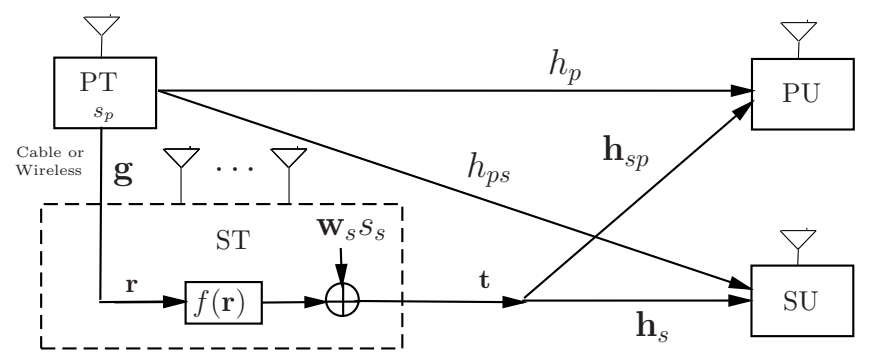

Fig. 1. Energy and information cooperation in cognitive radio.

We consider cooperation between a primary system and a secondary system in cognitive radio networks, as depicted in Fig. 1 The primary system consists of a primary transmitter (PT) and a primary user (PU), while the secondary system has a secondary transmitter (ST) who serves a secondary user (SU). All terminals have a single antenna except that the ST has $N$ antennas. The PT intends to send $s_{p}$ to the PU while the ST transmits signal $s_{s}$ to the SU with appropriate power. We consider a scenario that the primary link is in outage status when its rate demand cannot be met via the direct link, thus it becomes necessary for the PT to cooperate with the ST in order to meet the PU's QoS requirement. Without loss of generality, we assume the communication duration $T$ is normalized to be unity.

Some common system parameters are introduced as follows. $h_{p}, \mathbf{h}_{s}, \mathbf{h}_{s p}$ and $h_{p s}$ are used to denote the PT-PU, ST-SU, ST-PU and PT-SU channels, respectively. The PT is connected to the ST either via cable or wireless channel g. The PT's total energy (or average transmit power) is $P_{p}$ and the PU's rate requirement is $r_{p} \mathrm{bps} / \mathrm{Hz}$. The $\mathrm{ST}$ itself has an initial total energy of $P_{s 0}$ and further receives/harvests energy from the PT. All channels and noise elements are assumed to be statistically independent of each other. We assume that global perfect channel state information (CSI) is available at the ST. After the ST receives both information and energy from the PT, it processes the primary signal, harvests energy then uses the harvested energy together with its own energy, to serve the SU and relay the signal to the PU. Amplify-and-forward (AF) relaying protocol is employed by the ST.

We explicitly consider two components in the noise received at a terminal: one is the received thermal noise and the other is due to RF to baseband conversion, both are modeled as zero-mean additive white Gaussian noise (AWGN) with variances of $N_{0}$ and $N_{C}$ respectively. Assuming that they are independent, we may consider both types of noise if possible and define the combined received noise power as $\tilde{N}_{0}=N_{0}+N_{C}$.

There are different approaches that facilitate the information and energy transfer from the PT to the ST. In the sequel, we will introduce three specific cooperation schemes and find their optimal as well as low-complexity solutions. For fairness, the same amount of energy (for both the PT and the ST) is used in all schemes.

\section{IDEAL PRIMARY-COGNITIVE COOPERATION}

\section{A. System Model and Problem Formulation}

We first look into the ideal cooperation between the PT and the ST for information and energy transfer, where the ST has non-causal information about the primary signal and obtains energy from the PT via reliable backhaulling, for instance, cable. Note that although in practice, this cooperation scheme is either too difficult or too costly to implement, it provides a performance upper bound for practical cooperation protocols.

Since the primary message $s_{p}\left(\mathrm{E}\left[\left|s_{p}\right|^{2}\right]=1\right)$ is noncausally known at the ST, it can employ the DPC technique to encode the primary signal and superimpose its own secondary signal $s_{s}\left(\mathrm{E}\left[\left|s_{s}\right|^{2}\right]=1\right)$ such that no primary interference is introduced at the SU1. The received signal at the PU is

$$
y_{p}=\left(\sqrt{(1-\beta) P_{p}} h_{p}+\mathbf{h}_{s p}^{\dagger} \mathbf{w}_{p}\right) s_{p}+\mathbf{h}_{s p}^{\dagger} \mathbf{w}_{s} s_{s}+n_{p},
$$

where $\mathbf{w}_{p}$ is the beamforming vector used by the ST to forward the primary signal, and $n_{p} \in \mathcal{C N}\left(0, \tilde{N}_{0}\right)$ is the combined received noise at the PU. $\beta P_{p}(0 \leq \beta \leq 1)$ denotes the amount of energy transferred to the $\mathrm{ST}$ and received as $\eta \beta P_{p}$, where $\eta$ is the efficiency of energy transfer. The ST then has the total power of $P_{s 0}+\eta \beta P_{p}$ to serve both the PU and the SU.

Due to the use of DPC, the SU receives

$$
y_{s}=\mathbf{h}_{s}^{\dagger} \mathbf{w}_{s} s_{s}+n_{s}
$$

where $n_{s} \in \mathcal{C} \mathcal{N}\left(0, \tilde{N}_{0}\right)$ is the combined received noise at the SU. Then the primary and secondary received signal to interference-plus-noise ratios (SINRs), are, respectively,

$$
\Gamma_{p}=\frac{\left|\sqrt{(1-\beta) P_{p}} h_{p}+\mathbf{h}_{s p}^{\dagger} \mathbf{w}_{p}\right|^{2}}{\left|\mathbf{h}_{s p}^{\dagger} \mathbf{w}_{s}\right|^{2}+\tilde{N}_{0}}, \text { and } \Gamma_{s}=\frac{\left|\mathbf{h}_{s}^{\dagger} \mathbf{w}_{s}\right|^{2}}{\tilde{N}_{0}}
$$

It is easy to see that the optimal $\mathbf{w}_{p}$ admits the form $\mathbf{w}_{p}=$ $\sqrt{q_{p}} \frac{\mathbf{h}_{s p}}{\left\|\mathbf{h}_{s p}\right\|} e^{j \theta}$, where $\theta$ is chosen for coherent reception and $q_{p} \triangleq\left\|\mathbf{w}_{p}\right\|^{2}$. As a result, the achievable PU rate is

$R_{p}=\log _{2}\left(1+\gamma_{p}\right)=\log _{2}\left(1+\frac{\left(\sqrt{(1-\beta) P_{p}} h_{p}+\sqrt{q_{p}}\left\|\mathbf{h}_{s p}\right\|\right)^{2}}{\left|\mathbf{h}_{s p}^{\dagger} \mathbf{w}_{s}\right|^{2}+\tilde{N}_{0}}\right)$.

\footnotetext{
${ }^{1}$ Note that the ST can also pre-cancel the interference at the PU caused by its own secondary signal, however, this could lead to performance degradation because the SU will receive interference from both the PT and the ST.
} 
The problem of maximizing the SU rate subject to PU rate and ST power constraints is written as

$$
\begin{aligned}
\max _{\mathbf{w}_{s}, q_{p}, \beta} & \left|\mathbf{h}_{s}^{\dagger} \mathbf{w}_{s}\right| \\
\text { s.t. } & \frac{\left(\sqrt{(1-\beta) P_{p}}\left|h_{p}\right|+\sqrt{q_{p}}\left\|\mathbf{h}_{s p}\right\|\right)^{2}}{\left|\mathbf{h}_{s p}^{\dagger} \mathbf{w}_{s}\right|^{2}+\tilde{N}_{0}} \geq 2^{r_{p}}-1, \\
& \left\|\mathbf{w}_{s}\right\|^{2}+q_{p} \leq P_{s 0}+\beta \eta P_{p} \\
& 0 \leq \beta \leq 1, q_{p}>0
\end{aligned}
$$

\section{B. Feasibility}

Before solving (5), in Proposition 1 we first give the condition under which it is feasible and the proof is given in Appendix A

Proposition 1: Problem (5) is feasible if and only if the PU rate requirement $r_{p}$ is not larger than

$$
R_{p, \max } \triangleq\left\{\begin{array}{c}
\log _{2}\left(1+\frac{\left(\sqrt{P_{p}}\left|h_{p}\right|+\sqrt{P_{s 0}}\left\|\mathbf{h}_{s p}\right\|\right)^{2}}{\tilde{N}_{0}}\right), \\
\quad \text { if } P_{p} \eta^{2}\left\|\mathbf{h}_{s p}\right\|^{2}<P_{s 0}\left|h_{p}\right|^{2} ; \\
\log _{2}\left(1+\frac{P_{p} \eta+P_{s 0}}{\eta^{2}\left\|\mathbf{h}_{s p}\right\|^{2}+\eta\left|h_{p}\right|^{2}} \frac{\left(\left|h_{p}\right|^{2}+\eta\left\|\mathbf{h}_{s p}\right\|^{2}\right)^{2}}{\tilde{N}_{0}}\right), \\
\text { otherwise. }
\end{array}\right.
$$

According to Proposition 1, when $P_{p} \eta^{2}\left\|\mathbf{h}_{s p}\right\|^{2}<P_{s 0}\left|h_{p}\right|^{2}$ which means that the transferred power from the PT to the ST cannot bring sufficient performance gain, no energy transfer is needed. This may happen when primary power is limited, the secondary power is abundant, or the efficiency of the power transfer is too low, etc.

\section{Simplified Characterization of (5)}

Problem (5) is equivalent to the following convex problem:

$$
\begin{array}{ll}
\max _{\mathbf{w}_{s}, \mathbf{v}} & \operatorname{Re}\left(\mathbf{h}_{s}^{\dagger} \mathbf{w}_{s}\right) \\
\text { s.t. } & \left(\mathbf{g}^{\mathrm{T}} \mathbf{v}\right)^{2} \geq\left(2^{r_{p}}-1\right)\left(\left|\mathbf{h}_{s p}^{\dagger} \mathbf{w}_{s}\right|^{2}+\tilde{N}_{0}\right), \\
& \left\|\mathbf{w}_{s}\right\|^{2}+\mathbf{v}^{\mathrm{T}} \mathbf{D} \mathbf{v} \leq P_{s 0}+\eta P_{p}, \\
& \mathbf{v} \geq \mathbf{0},[\mathbf{v}]_{1} \leq 1 .
\end{array}
$$

where $\quad \mathbf{g}^{\mathrm{T}}=\left[\sqrt{P_{p}}\left|h_{p}\right|,\left\|\mathbf{h}_{s p}\right\|\right], \quad \mathbf{D}=$ $\operatorname{diag}\left(\eta P_{p}, 1\right), \mathbf{v} \triangleq\left[\sqrt{1-\beta}, \sqrt{q_{p}}\right]$. Optimization problem in (7) is a second order cone problem (SOCP) and is convex. It can be solved very efficiently. The steps of recasting (5) to the convex problem (7) are given in Appendix B

Although problem (5) can be manipulated as a convex problem, it does not offer much insight into the structure of the solution. In the following, from the characterization of the optimal solution structure, we identify very efficient solutions. To this end, we first show that at the optimality point, the two constraints in (5) are active. If the power constraint is not active at the optimality point, one can update the beamforming vector $\mathbf{w}_{s}$ to $\mathbf{w}_{s}^{\prime}=\mathbf{w}_{s}+\tau \Pi_{\mathbf{h}_{s p}}^{\perp} \mathbf{h}_{s}$ with $\tau$ being a very small scalar, which increases the objective value while keeping the first constraint unchanged. This contradicts the optimality point assumption. Then if the first constraint is not active, one can decrease the value of $q_{p}$ and increase the value of $\beta$ such that the first constraint is active but the power constraint is not active. This leads us back to the previous case and a contradiction of the optimality assumption results. Hence at the optimality point, the two constraints are always active. The main result of the simplified optimization problem is given in Proposition 2 and its proof is provided in Appendix $\mathrm{C}$

Proposition 2: Problem (5) is equivalent to the problem (8) at the top of next page.

Problem (8) is not convex in general, so we propose to find its optimal solution via 2-D search.

We illustrate the feasibility region in Figure 2 where randomly chosen channel realizations and system settings are given by $\mathbf{h}_{s}=[-0.0823+$ $1.3427 i,-0.6438-0.4291 i, 0.4338-0.2197 i]^{T}$, $\mathbf{h}_{s p}=[0.5345-0.8716 i, 0.2872-0.4043 i, 0.0951-0.3264 i]^{T}$, $h_{p}=-0.4692+0.8665 i, \gamma_{0}=5, P_{p}=P_{s p}=10, \eta=0.8$ and $N=3$. The feasibility region of two target SINR values are shown. Each blue ring circle is composed of tuples of $\left(\left|\mathbf{h}_{s p}^{\dagger} \mathbf{w}_{s}\right|,\left|\mathbf{h}_{s}^{\dagger} \mathbf{w}_{s}\right|^{2}\right)$ and is obtained by varying $\mathbf{w}_{s}$ and keeping $q_{p}$ and $\beta$ fixed. When $q_{p}$ increases and $\beta$ fixed, constraint 5b on $\mathbf{h}_{s p}^{\dagger} \mathbf{w}_{s}$ is more relaxed. This is illustrated by the red lines, each line corresponding to a given pair $q_{p}, \beta$. When $q_{p}$ increases, the red line moves up. Similarly, when $q_{p}$ increases and $\beta$ fixed, (5c) becomes more strict and thus the blue region shrinks. Similar behavior can be observed if $\beta$ varies and $q_{p}$ is kept constant. The feasibility region is the blue region under the corresponding red line. Hence, there is a conflict between constraints (5b) and (5c). In Figure 2, the black cross marker shows the optimal point for each given tuple of $\left(q_{p}, \beta\right)$. The optimal point is the black cross with the largest $\mathrm{x}$-coordinate. In Figure 3, we collect the optimal points (shown in black crosses in both Figure 2 and 3 ) for each paired value of $\left(q_{p}, \beta\right)$. Out of these optimal points of each realization of $\left(q_{p}, \beta\right)$, we mark the optimal point of the whole set as a red square. If the zero-forcing scheme is implemented, the achievable points by definition are always on the $\mathrm{x}$-axis and the optimal point is marked as a blue triangle in Figure 3

\section{ZF Solution}

Here we study a suboptimal yet closed-form solution with $\mathrm{ZF}$ constraint on the interference power from the ST to the PU, i.e., $\mathbf{h}_{s p}^{\dagger} \mathbf{w}_{s}=0$. To satisfy this, the beamforming vector $\mathbf{w}_{s}$ is chosen as

$$
\mathbf{w}_{s, Z F}=\sqrt{q_{s}} \frac{\left(\mathbf{I}-\frac{\mathbf{h}_{s p} \mathbf{h}_{s p}^{\dagger}}{\left\|\mathbf{h}_{s p}\right\|^{2}}\right) \mathbf{h}_{s}}{\left\|\left(\mathbf{I}-\frac{\mathbf{h}_{s p} \mathbf{h}_{s p}^{\dagger}}{\left\|\mathbf{h}_{s p}\right\|^{2}}\right) \mathbf{h}_{s}\right\|},
$$

and the resulting SU channel gain is

$$
\left|\mathbf{w}_{s, Z F}^{\dagger} \mathbf{h}_{s}\right|^{2}=q_{s}\left\|\mathbf{h}_{s}\right\|^{2}\left(1-\delta^{2}\right), \delta^{2} \triangleq \frac{\left|\mathbf{h}_{s p}^{\dagger} \mathbf{h}_{s}\right|^{2}}{\left\|\mathbf{h}_{s p}\right\|^{2}\left\|\mathbf{h}_{s}\right\|^{2}}
$$




$$
\begin{aligned}
\max _{\beta, q_{p}} & \sqrt{\frac{\left|\sqrt{(1-\beta) P_{p}} \frac{\left|h_{p}\right|}{\left\|\mathbf{h}_{s p}\right\|}+\sqrt{q_{p}}\right|^{2}}{2^{r_{p}}-1}-\frac{\tilde{N}_{0}}{\left\|\mathbf{h}_{s p}\right\|^{2}}\left\|\Pi_{\mathbf{h}_{s p}} \mathbf{h}_{s}\right\|} \\
& +\sqrt{\left(P_{s 0}+\beta \eta P_{p}\right)-q_{p}-\frac{\left|\sqrt{(1-\beta) P_{p}} \frac{\left|h_{p}\right|}{\left\|\mathbf{h}_{s p}\right\|}+\sqrt{q_{p}}\right|^{2}}{2^{r_{p}}-1}-\frac{\tilde{N}_{0}}{\left\|\mathbf{h}_{s p}\right\|^{2}}\left\|\Pi_{\mathbf{h}_{s p}}^{\perp} \mathbf{h}_{s}\right\|} \\
\text { s.t. } & 0 \leq \beta \leq 1, q_{p}>0 .
\end{aligned}
$$

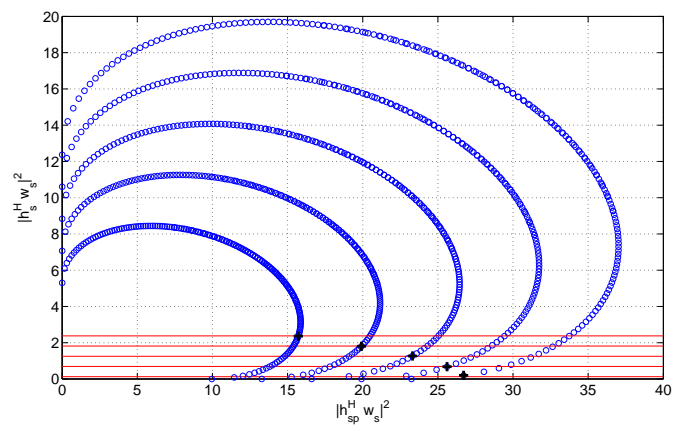

Fig. 2. The channel power region of $\left|\mathbf{h}_{s}^{\dagger} \mathbf{w}_{s}\right|^{2}$ against $\left|\mathbf{h}_{s p}^{\dagger} \mathbf{w}_{s}\right|^{2} .(\cdot)^{H}$ represents Hermitian operation in the figure and is denoted as $(\cdot)^{\dagger}$ in the main text. The red lines show the constraint moving up when $q_{p}$ increases and $\beta$ fixed. At the same time, the channel power region, shown in blue, shrinks. The feasibility region is the blue region under the corresponding red line. Within the feasibility regions, the point with the maximum $\mathrm{x}$-coordinate is marked with a black cross.

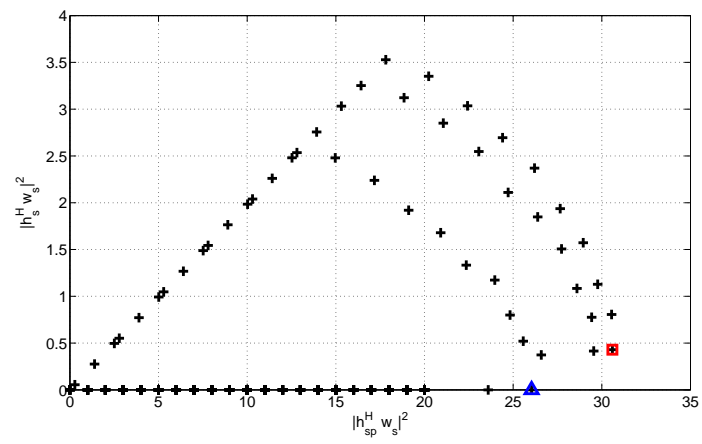

Fig. 3. The optimal point for each pair of values of $\left(q_{p}, \beta\right)$ is collected and shown in black crosses. The optimal point that attains the largest value of $\left|h_{s p}^{\dagger} \mathbf{w}_{s}\right|^{2}$, is marked as a red square. When the zero-forcing scheme in Section III-D is used, the optimal point is marked as a blue triangle.

As a result, the optimization problem is formulated as

$$
\begin{aligned}
\max _{q_{s}, q_{p}, \beta} & q_{s} \\
\text { s.t. } & \frac{\left(\sqrt{(1-\beta) P_{p}}\left|h_{p}\right|+\sqrt{q_{p}}\left\|\mathbf{h}_{s p}\right\|\right)^{2}}{\tilde{N}_{0}} \geq 2^{r_{p}}-1, \\
& q_{s}+q_{p} \leq P_{s 0}+\beta \eta P_{p}, \\
& 0 \leq \beta \leq 1, q_{s} \geq 0, q_{p} \geq 0 .
\end{aligned}
$$

Due to the fact that both constraints (12) and (13) should hold with equality, $q_{s}$ can be expressed as

$$
\begin{aligned}
& q_{s}=P_{s 0}+\beta \eta P_{p}-q_{p} \\
& =P_{s 0}+\beta P_{p}\left(\eta+\frac{\left|h_{p}\right|^{2}}{\left\|\mathbf{h}_{s p}\right\|^{2}}\right)- \\
& \frac{\left(2^{r_{p}}-1\right) \tilde{N}_{0}+P_{p}\left|h_{p}\right|^{2}-2 \sqrt{\left(2^{r_{p}}-1\right) \tilde{N}_{0}} \sqrt{(1-\beta) P_{p}}\left|h_{p}\right|}{\left\|\mathbf{h}_{s p}\right\|^{2}} .
\end{aligned}
$$

By setting the first-order derivative of 16 to be zero, we can derive the optimal $\beta$ as

$$
\beta^{*}=\left[1-\frac{\left(2^{r_{p}}-1\right) \tilde{N}_{0}}{P_{p}\left(\left|h_{p}\right|+\frac{\eta\left\|\mathbf{h}_{s p}\right\|^{2}}{\left|h_{p}\right|}\right)^{2}}\right]_{0}^{1} .
$$

The expression (16) verifies the intuition that the optimal $\beta$ is an increasing function of $P_{p},\left\|\mathbf{h}_{s p}\right\|^{2}$ and $\eta$. If the PT has abundant power, then it is more likely to transfer energy to the ST. On the other hand, if the ST-PU link is weak or the efficiency of energy transfer is low, it is not worth transferring too much energy to the ST. There is an interesting observation about $\left|h_{p}\right|$. If $\left|h_{p}\right|$ is close to zero, $\beta$ approaches 1 , which means that the primary system relies on the ST to forward its signal, therefore transfers all its energy to the ST. As the primary channel becomes better or $\left|h_{p}\right|$ increases but is below the threshold $\sqrt{\eta}\left\|\mathbf{h}_{s p}\right\|, \beta$ is a decreasing function of $\left|h_{p}\right|$; once $\left|h_{p}\right|$ exceeds the threshold $\sqrt{\eta}\left\|\mathbf{h}_{s p}\right\|, \beta$ becomes an increasing function of $\left|h_{p}\right|$ and this is because the primary channel is good enough therefore the primary system can help the secondary transmission.

The channel power values achieved by the ZF solution achieves are shown in Figure 3. The corresponding optimal solution is marked blue. The ZF solution is simple and we observe that its performance is quite good in this example.

The implementation of the ideal cooperation requires cable and common energy source, as well as signal processing and coding capabilities for DPC. In the following two sections, we consider two practical energy and information cooperation schemes. We assume that the ST adopts the AF relaying protocol to forward the primary signals instead of processing non-causal primary information.

\section{Power Splitting Cooperation - System Model AND OPTIMIZATION}

\section{A. System Model and Problem Setting}

In this section, we assume the ST first listens to the primary transmission via the channel $\mathrm{g}$ then forwards it to the PU, 


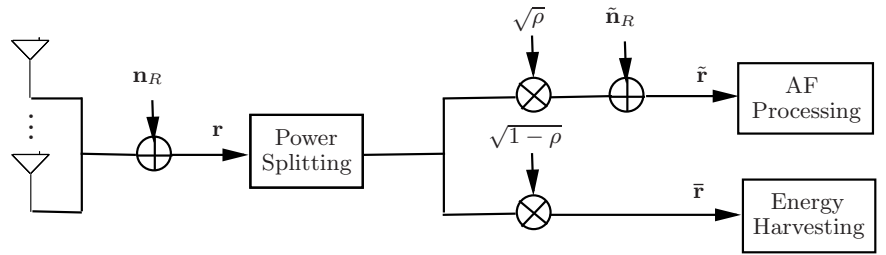

Fig. 4. The power splitting EH technique at the ST.

therefore two channel phases are required to complete the communications. In Phase I, the PT broadcasts its data $s_{p}$ with power $2 P_{p}$ where the factor 2 is because the PT only transmits during the first half duration, then the received signals at the $\mathrm{PU}$ and the ST are, respectively,

$$
y_{p 1}=\sqrt{2 P_{p}} h_{p} s_{p}+n_{p 1}, \quad \text { and } \mathbf{r}=\sqrt{2 P_{p}} \mathbf{g} s_{p}+\mathbf{n}_{R},
$$

where $n_{p 1} \in \mathcal{C N}\left(0, \tilde{N}_{0}\right)$ is the combined noise at the PU while $\mathbf{n}_{R} \in \mathcal{C N}\left(\mathbf{0}, N_{0} \mathbf{I}\right)$ is the thermal noise received at $\mathrm{ST}$, respectively.

To forward primary information as well as harvest RF energy at the ST, the practical power splitting technique [17 is used, which is depicted in Fig. 4 and works as follows. The ST splits the RF signal into two portions: one for forwarding to the PU after AF processing and the other for harvesting energy, with relative power of $\rho$ and $1-\rho$, respectively. The signal for AF processing will be converted from the RF to the baseband, and this results in the received signal

$$
\tilde{\mathbf{r}}=\sqrt{\rho} \mathbf{r}+\tilde{\mathbf{n}}_{R},
$$

where $\tilde{\mathbf{n}}_{R} \sim \mathcal{C N}\left(\mathbf{0}, N_{C} \mathbf{I}\right)$ is the complex AWGN during the $\mathrm{RF}$ to baseband conversion. The ST processes the received signal and produces $f(\mathbf{r})=\mathbf{A} \tilde{\mathbf{r}}$. Without loss of optimality, it has been shown that the optimal $\mathbf{A}$ has the structure of $\mathbf{A}=\mathbf{w}_{p} \mathbf{g}^{\dagger}$ according to [14], where $\mathbf{w}_{p}$ is a new transmit beamforming vector to be optimized. This is also intuitive because there is a single primary data stream, the best reception strategy for the ST is to use maximal ratio combining (MRC).

The signal for EH is simply

$$
\overline{\mathbf{r}}=\sqrt{1-\rho} \mathbf{r}=\sqrt{1-\rho}\left(\mathbf{g} \sqrt{2 P_{p}} s_{p}+\mathbf{n}_{R}\right) .
$$

Assuming the energy transfer efficiency of $\eta$, the amount of the harvested energy is

$$
P_{E H}=\frac{\eta(1-\rho)\left(2 P_{p}\|\mathbf{g}\|^{2}+N_{0}\right)}{2} .
$$

Therefore, the ST will have a total transmit power of $2 P_{s 0}+$ $\eta(1-\rho)\left(2 P_{p}\|\mathbf{g}\|^{2}+N_{0}\right)$ where the factor 2 is due to the fact that the ST only transmits signals in the second half of the communication time.

In Phase II, the ST superimposes the relaying signal $f(\mathbf{r})$ with its own data $s_{s}$ using the cognitive beamforming vector $\mathbf{w}_{s}$, then transmits it to both the PU and the SU. Note that DPC is not used at the ST. In this phase, the PT remains idle.

The ST's transmit signal is

$$
\begin{aligned}
\mathbf{t} & =\mathbf{w}_{s} s_{s}+\mathbf{w}_{p} \mathbf{g}^{\dagger} \tilde{\mathbf{r}} \\
& =\mathbf{w}_{s} s_{s}+\sqrt{2 \rho P_{p}} \mathbf{w}_{p}\|\mathbf{g}\|^{2} s_{p}+\sqrt{\rho} \mathbf{w}_{p} \mathbf{g}^{\dagger} \mathbf{n}_{R}+\mathbf{w}_{p} \mathbf{g}^{\dagger} \tilde{\mathbf{n}}_{R},
\end{aligned}
$$

with average power

$p_{R}=\mathrm{E}\|\mathbf{t}\|^{2}=\left\|\mathbf{w}_{s}\right\|^{2}+\left(2 P_{p} \rho\|\mathbf{g}\|^{4}+\rho\|\mathbf{g}\|^{2} N_{0}+\|\mathbf{g}\|^{2} N_{C}\right)\left\|\mathbf{w}_{p}\right\|^{2}$.

The received signal at the $\mathrm{SU}$ is

$$
\begin{aligned}
y_{s}= & \mathbf{h}_{s}^{\dagger} \mathbf{t}+n_{s} \\
= & \mathbf{h}_{s}^{\dagger} \mathbf{w}_{s} s_{s}+\sqrt{\rho} \mathbf{h}_{s}^{\dagger} \mathbf{w}_{p}\|\mathbf{g}\|^{2} s_{p}+\sqrt{\rho} \mathbf{h}_{s}^{\dagger} \mathbf{w}_{p} \mathbf{g}^{\dagger} \mathbf{n}_{R} \\
& +\mathbf{h}_{s}^{\dagger} \mathbf{w}_{p} \mathbf{g}^{\dagger} \tilde{\mathbf{n}}_{R}+n_{s},
\end{aligned}
$$

where $n_{s} \in \mathcal{C N}\left(0, \tilde{N}_{0}\right)$ is the combined noise at the SU. The received SINR at SU is then expressed as

$$
\Gamma_{s}=\frac{\left|\mathbf{h}_{s}^{\dagger} \mathbf{w}_{s}\right|^{2}}{\left(2 P_{p} \rho\|\mathbf{g}\|^{4}+\rho\|\mathbf{g}\|^{2} N_{0}+\|\mathbf{g}\|^{2} N_{C}\right)\left|\mathbf{h}_{s}^{\dagger} \mathbf{w}_{p}\right|^{2}+\tilde{N}_{0}},
$$

and the achievable SU rate is $R_{s}=\frac{1}{2} \log _{2}\left(1+\Gamma_{s}\right)$ where the factor $\frac{1}{2}$ arises due to the two orthogonal channel uses. The received signal at the $\mathrm{PU}$ is

$$
\begin{aligned}
y_{p 2}= & \mathbf{h}_{s p}^{\dagger} \mathbf{t}+n_{p 2} \\
= & \mathbf{h}_{s p}^{\dagger} \mathbf{w}_{s} s_{s}+\rho \mathbf{h}_{s p}^{\dagger} \mathbf{w}_{p}\|\mathbf{g}\|^{2} s_{p}+\rho \mathbf{h}_{s p}^{\dagger} \mathbf{w}_{p} \mathbf{g}^{\dagger} \mathbf{n}_{R} \\
& +\mathbf{h}_{s p}^{\dagger} \mathbf{w}_{p} \mathbf{g}^{\dagger} \tilde{\mathbf{n}}_{R}+n_{p 2},
\end{aligned}
$$

where $n_{p 2} \in \mathcal{C N}\left(0, \tilde{N}_{0}\right)$ is the combined noise at the PU during Phase II.

Applying the MRC strategy to $y_{p 1}$ and $y_{p 2}$, the received SINR of the PU is the sum of two channel uses, and consequently, the achievable PU rate is

$$
\begin{aligned}
& R_{p}=\frac{1}{2} \log _{2}\left(1+\frac{2 P_{p}\left|h_{p}\right|^{2}}{\tilde{N}_{0}}+\right. \\
& \left.\frac{2 P_{p} \rho\|\mathbf{g}\|^{4}\left|\mathbf{h}_{s p}^{\dagger} \mathbf{w}_{p}\right|^{2}}{\left|\mathbf{h}_{s p}^{\dagger} \mathbf{w}_{s}\right|^{2}+\left(\rho\|\mathbf{g}\|^{2} N_{0}+\|\mathbf{g}\|^{2} N_{C}\right)\left|\mathbf{h}_{s p}^{\dagger} \mathbf{w}_{p}\right|^{2}+\tilde{N}_{0}}\right) .
\end{aligned}
$$

Next we can formulate the problem of maximizing the SU rate $R_{s}$ subject to the PU's rate constraint $r_{p}$ and the ST's transmit power constraint $2\left(P_{s 0}+P_{E H}\right)$, by jointly optimizing the power splitting parameter $\rho$, the cognitive beamforming vector $\mathbf{w}_{s}$, and the forwarding beamforming vector $\mathbf{w}_{p}$. Using the monotonicity between the received SINR and the achievable rate, the optimization problem can be written as

$$
\begin{aligned}
\max _{\mathbf{w}_{s}, \mathbf{w}_{p}, \rho} & \frac{\left|\mathbf{h}_{s}^{\dagger} \mathbf{w}_{s}\right|^{2}}{\left(2 P_{p} \rho\|\mathbf{g}\|^{4}+\rho\|\mathbf{g}\|^{2} N_{0}+\|\mathbf{g}\|^{2} N_{C}\right)\left|\mathbf{h}_{s}^{\dagger} \mathbf{w}_{p}\right|^{2}+\tilde{N}_{0}}(27) \\
\text { s.t. } & \frac{\left|\mathbf{h}_{s p}^{\dagger} \mathbf{w}_{p}\right|^{2}}{\left|\mathbf{h}_{s p}^{\dagger} \mathbf{w}_{s}\right|^{2}+\tilde{N}_{0}} \geq \frac{\gamma_{p}^{\prime}}{\rho\|\mathbf{g}\|^{4}-\gamma_{p}^{\prime}\left(\rho\|\mathbf{g}\|^{2} N_{0}+\|\mathbf{g}\|^{2} N_{C}\right)}, \\
& \left\|\mathbf{w}_{s}\right\|^{2}+\left(2 P_{p} \rho\|\mathbf{g}\|^{4}+\rho\|\mathbf{g}\|^{2} N_{0}+\|\mathbf{g}\|^{2} N_{C}\right)\left\|\mathbf{w}_{p}\right\|^{2} \leq \\
& 2 P_{s 0}+\eta(1-\rho)\left(2 P_{p}\|\mathbf{g}\|^{2}+N_{0}\right), \\
& 0 \leq \rho \leq 1,
\end{aligned}
$$

where we have defined $\gamma_{p}^{\prime} \triangleq \frac{2^{2 r_{p}}-1}{2 P_{p}}-\frac{\left|h_{p}\right|^{2}}{\tilde{N}_{0}}$.

\section{B. Feasibility Check}

Before solving problem (27), we first investigate its feasibility, and this can be achieved by finding the maximum PU rate $R_{P}$ or equivalently $\gamma_{p}^{\prime}$. To achieve the maximum $\gamma_{p}^{\prime}$, we set $\mathbf{w}_{s}=\mathbf{0}$ then we have $\left\|\mathbf{w}_{p}\right\|^{2}=\frac{2 P_{s 0}+\eta(1-\rho)\left(2 P_{p}\|\mathbf{g}\|^{2}+N_{0}\right)}{2 P_{p} \rho\|\mathbf{g}\|^{4}+\rho\|\mathbf{g}\|^{2} N_{0}+\|\mathbf{g}\|^{2} N_{C}}$, 
and reach an optimization problem about $\rho$ below:

$\begin{aligned} \max _{\rho} & \frac{\rho\|\mathbf{g}\|^{4}}{\frac{\tilde{N}_{0}\left(2 P_{p} \rho\|\mathbf{g}\|^{4}+\rho\|\mathbf{g}\|^{2} N_{0}+\|\mathbf{g}\|^{2} N_{C}\right)}{\left\|\mathbf{h}_{s p}\right\|^{2}\left(2 P_{s 0}+\eta(1-\rho)\left(2 P_{p}\|\mathbf{g}\|^{2}+N_{0}\right)\right)}+\left(\rho\|\mathbf{g}\|^{2} N_{0}+\|\mathbf{g}\|^{2} N_{C}\right)} \\ \text { s.t. } & 0 \leq \rho \leq 1 .\end{aligned}$

The unique optimal $\rho^{*}$ can be computed in closed form, despite its complicated expression. For details, please see Appendix [D While $\rho^{*}$ corresponds to a maximum PU rate $R_{P}^{*}$, we can choose any rate smaller than $R_{P}^{*}$ in solving [27].

\section{The Optimal Solution}

Assuming problem 27) is feasible, we study how to find its optimal solution.

$\begin{array}{ccc}\text { By change of variable } & \mathbf{w}_{p} & := \\ \sqrt{2 P_{p} \rho\|\mathbf{g}\|^{4}+\rho\|\mathbf{g}\|^{2} N_{0}+\|\mathbf{g}\|^{2} N_{C}} \mathbf{w}_{p} & \text { we write }\end{array}$ in an equivalent but more compact form as

$$
\begin{aligned}
& \max _{\mathbf{w}_{s}, \mathbf{w}_{p}, \rho} \frac{\left|\mathbf{h}_{s}^{\dagger} \mathbf{w}_{s}\right|^{2}}{\left|\mathbf{h}_{s}^{\dagger} \mathbf{w}_{p}\right|^{2}+\tilde{N}_{0}} \\
& \text { s.t. } \quad \frac{\left|\mathbf{h}_{s p}^{\dagger} \mathbf{w}_{p}\right|^{2}}{\left|\mathbf{h}_{s p}^{\dagger} \mathbf{w}_{s}\right|^{2}+\tilde{N}_{0}} \geq \gamma_{p}^{\prime \prime}, \\
& \left\|\mathbf{w}_{s}\right\|^{2}+\left\|\mathbf{w}_{p}\right\|^{2} \leq 2 P_{s 0}+\eta(1-\rho)\left(2 P_{p}\|\mathbf{g}\|^{2}+N_{0}\right), \\
& 0 \leq \rho \leq 1 \text {, }
\end{aligned}
$$

where $\gamma_{p}^{\prime \prime} \triangleq \frac{\left(2 P_{p} \rho\|\mathbf{g}\|^{2}+\rho N_{0}+N_{C}\right) \gamma_{p}^{\prime}}{\rho\|\mathbf{g}\|^{2}-\gamma_{n}^{\prime}\left(\rho N_{0}+N_{C}\right)}$. We find the following lemma useful to solve (29).

Lemma 1: Consider a general maximization problem below:

$$
\begin{array}{cl}
\max _{\mathbf{w}_{1}, \mathbf{w}_{2}} & \frac{\left|\mathbf{h}_{2}^{\dagger} \mathbf{w}_{2}\right|^{2}}{\sigma^{2}+\left|\mathbf{h}_{2}^{\dagger} \mathbf{w}_{1}\right|^{2}} \\
\text { s.t. } & \frac{\left|\mathbf{h}_{1}^{\dagger} \mathbf{w}_{1}\right|^{2}}{\sigma^{2}+\left|\mathbf{h}_{1}^{\dagger} \mathbf{w}_{2}\right|^{2}} \geq \gamma_{1} \\
& \left\|\mathbf{w}_{1}\right\|^{2}+\left\|\mathbf{w}_{2}\right\|^{2} \leq P_{C},
\end{array}
$$

where $\mathbf{h}_{1}, \mathbf{h}_{2}$ are $N \times 1$ vectors and $\gamma_{1}, P_{C}, \sigma^{2}$ are positive scalars. Define $\zeta^{2} \triangleq \frac{\left|\mathbf{h}_{1}^{\dagger} \mathbf{h}_{2}\right|^{2}}{\left\|\mathbf{h}_{1}\right\|^{2}\left\|\mathbf{h}_{2}\right\|^{2}}$. Suppose (30) is feasible and its optimal objective value is $\gamma_{2}$, then $\gamma_{2}$ is uniquely determined by the following equation set:

$$
\begin{cases}\lambda_{1} & =\frac{\gamma_{1} \sigma^{2}\left(\sigma^{2}+\lambda_{2}\left\|\mathbf{h}_{2}\right\|^{2}\right)}{\left\|\mathbf{h}_{1}\right\|^{2}\left(\sigma^{2}+\lambda_{2}\left\|\mathbf{h}_{2}\right\|^{2}\left(1-\zeta^{2}\right)\right)} \\ \lambda_{2} & =\frac{\gamma_{2} \sigma^{2}\left(\sigma^{2}+\lambda_{1}\left\|\mathbf{h}_{1}\right\|^{2}\right)}{\left\|\mathbf{h}_{2}\right\|^{2}\left(\sigma^{2}+\lambda_{1}\left\|\mathbf{h}_{1}\right\|^{2}\left(1-\zeta^{2}\right)\right)} \\ \lambda_{1}+\lambda_{2} & =P_{C}\end{cases}
$$

where $\lambda_{1}, \lambda_{2}$ are dual variables.

Proof: See Appendix A in [16].

Given $\rho$, using Lemma 1 the dual variables $\left(\lambda_{1}, \lambda_{2}\right)$ of 29 are identified. Then the optimal $\mathbf{w}_{s}, \mathbf{w}_{p}$ can be expressed as

$$
\left\{\begin{array}{l}
\mathbf{w}_{1}=\sqrt{p_{1}} \frac{\left(\sigma^{2} \mathbf{I}+\lambda_{2} \mathbf{h}_{2} \mathbf{h}_{2}^{\dagger}\right)^{-1} \mathbf{h}_{1}}{\left\|\left(\sigma^{2} \mathbf{I}+\lambda_{2} \mathbf{h}_{2} \mathbf{h}_{2}^{\dagger}\right)^{-1} \mathbf{h}_{1}\right\|}, \\
\mathbf{w}_{2}=\sqrt{p_{2}} \frac{\left(\sigma^{2} \mathbf{I}+\lambda_{1} \mathbf{h}_{1} \mathbf{h}_{1}^{\dagger}\right)^{-1} \mathbf{h}_{2}}{\left\|\left(\sigma^{2} \mathbf{I}+\lambda_{1} \mathbf{h}_{1} \mathbf{h}_{1}^{\dagger}\right)^{-1} \mathbf{h}_{2}\right\|},
\end{array}\right.
$$

where the downlink power $p_{1}, p_{2}$ can be found using the uplink-downlink duality [30]. Then the optimal solution to (29) can be derived by performing 1-D optimization of $\rho$. To efficiently find the optimal $\rho$, we characterize its feasible range in Appendix E

\section{Closed-form ZF Solutions}

In order to gain more insight into the system parameters, we study the ZF solution which allows a closed-form solution. According to the ZF criterion, there should be no interference between the primary and the secondary transmission, which requires that $\mathbf{h}_{s}^{\dagger} \mathbf{w}_{p}=\mathbf{h}_{s p}^{\dagger} \mathbf{w}_{s}=0$. The ZF solution to $\mathbf{w}_{s}$ has been given in (9) and similarly, the ZF solution to $\mathbf{w}_{p}$ can be derived as

$$
\mathbf{w}_{p, Z F}=\sqrt{q_{p}} \frac{\left(\mathbf{I}-\frac{\mathbf{h}_{s} \mathbf{h}_{s}^{\dagger}}{\left\|\mathbf{h}_{s}\right\|^{2}}\right) \mathbf{h}_{s p}}{\left\|\left(\mathbf{I}-\frac{\mathbf{h}_{s} \mathbf{h}_{s}^{\dagger}}{\left\|\mathbf{h}_{s}\right\|^{2}}\right) \mathbf{h}_{s p}\right\|}
$$

with the resulting channel gain to the PU being $\left|\mathbf{w}_{p, Z F}^{\dagger} \mathbf{h}_{s p}\right|^{2}=$ $q_{p}\left\|\mathbf{h}_{s p}\right\|^{2}\left(1-\delta^{2}\right)$.

Therefore problem 29) reduces to

$$
\begin{aligned}
\max _{q_{p}, q_{s}, \rho} & q_{s} \\
\text { s.t. } & \frac{q_{p}\left\|\mathbf{h}_{s p}\right\|^{2}\left(1-\delta^{2}\right)}{\tilde{N}_{0}} \geq \gamma_{p}^{\prime \prime}, \\
& q_{s}+q_{p} \leq 2 P_{s 0}+\eta(1-\rho)\left(2 P_{p}\|\mathbf{g}\|^{2}+N_{0}\right), \\
& 0 \leq \rho \leq 1, q_{p} \geq 0, q_{s} \geq 0 .
\end{aligned}
$$

The optimal $\rho_{z f}^{*}$ is given by

$$
\rho_{z f}^{*}=\left[\frac{\frac{\sqrt{\gamma_{p}^{\prime} N_{C} \tilde{N}_{0}}}{\sqrt{\left\|\mathbf{h}_{s p}\right\|^{2}\left(1-\delta^{2}\right)}} \sqrt{\gamma_{p}^{\prime}+\frac{\left(\|\mathbf{g}\|^{2}-\gamma_{p}^{\prime} N_{0}\right)}{\eta\left(2 P_{p}\|\mathbf{g}\|^{2}+N_{0}\right)}}+\gamma_{p}^{\prime} N_{C}}{\|\mathbf{g}\|^{2}-\gamma_{0}^{\prime} N_{C}}\right]_{0}^{1} .
$$

Derivation of the solution and its feasible range are given in Appendix E

We can draw some insights from (35) on $\rho_{z f}^{*}$ :

- It increases with $\gamma_{p}^{\prime}=\frac{2^{2 r_{p}}-1}{2 P_{p}}-\frac{\left|h_{p}\right|^{2}}{\tilde{N}_{0}}$, or decreases with $P_{p}$ and $\left|h_{p}\right|^{2}$, which means when the primary channel is good or power is abundant, PU rate is easy to satisfy, and thus the PT can transfer more energy to the ST.

- It decreases with $\eta$ and $\left\|\mathbf{h}_{s p}\right\|^{2}$, which means if the efficiency of energy transfer is low or the ST-PU channel is weak, more received signal is used for information decoding. This is different from the ideal cooperation case where the primary signal is non-causally known at the ST.

To illustrate the solutions, the achievable SU rates against the power splitting parameter $\rho$ for the optimal solution and ZF solution are compared in Fig. 5 for a specific channel realization $N=3,\left|h_{p}\right|^{2}=0.0127, \mathrm{~g}=[0.8113-$ $1.5579 i 0.4228-0.4039 i-0.9060+0.1513 i]^{T}, \mathbf{h}_{s}=$ $[0.6664+0.2165 i 0.0663-0.8290 i-0.7936-0.6795 i]^{T}$ and $\mathbf{h}_{s p}=[-0.4623-0.6364 i-0.8693-0.2020 i-0.1916-$ $0.3270 i]^{T}$. The primary power is set to $P_{p}=10 \mathrm{~dB}$ and the ST's own power is $P_{s 0}=0 \mathrm{~dB}$. The PU's target rate is 2.6 $\mathrm{bps} / \mathrm{Hz}$. All noise variance is normalized to one. It is clearly seen that the feasible range of the optimal solution includes that of the ZF solution as a subset. The optimal SU rate is higher than double of the ZF SU rate. 


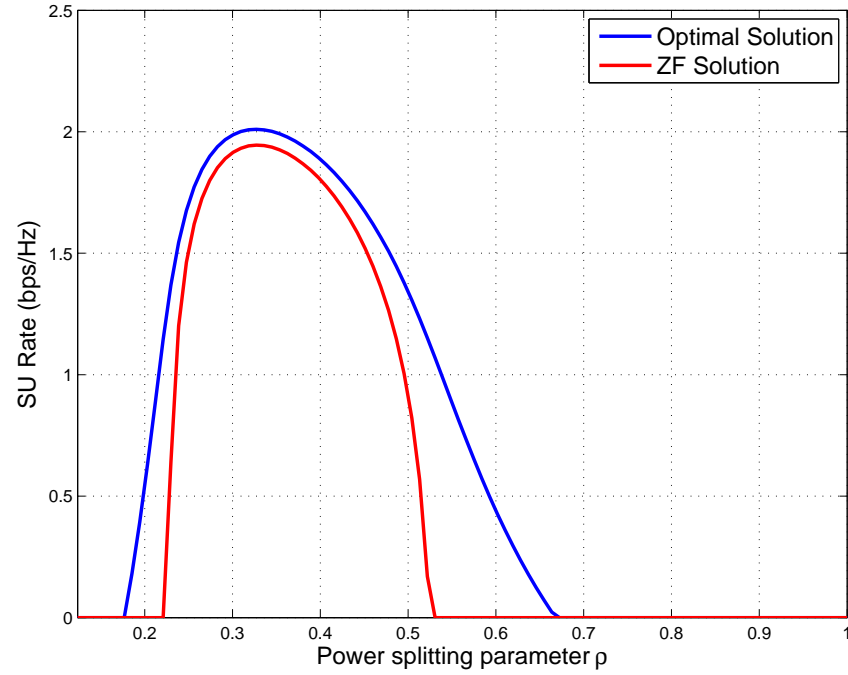

Fig. 5. SU rate vs $\rho$ for power splitting.

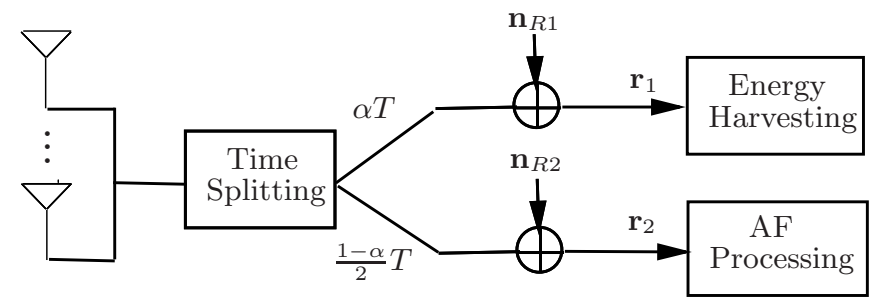

Fig. 6. The time splitting EH technique at the ST.

\section{Time Splitting Cooperation - System Model AND OPTIMIZATION}

\section{A. System Model and Problem Setting}

In this section, we study the optimization of a three-phase time-splitting cooperation protocol where the time-splitting EH is illustrated in Fig. 6 The PT first uses a dedicated time slot with a duration of $\alpha(0 \leq \alpha \leq 1)$ to transfer energy to the ST. In the remaining two equal-time phases with duration of $\frac{1-\alpha}{2}$, the PT transmits data to the ST then the ST forwards the primary signal to the PU and serves its own SU. The PT can adjust its transmit power in the two phases as long as it does not exceed the peak power constraint $P_{\max }$. The signal model is described below.

In Phase I, the PT sends signal $s_{p 1}$ with average power $P_{p 1}$ to both the ST for energy harvesting and the PU for information decoding. The received signal at the ST is

$$
\mathbf{r}_{1}=\mathbf{g} s_{p 1}+\mathbf{n}_{R 1},
$$

where $\mathbf{n}_{R 1} \in \mathcal{C N}\left(\mathbf{0}, \tilde{N}_{0} \mathbf{I}\right)$ is the AWGN received at the ST. The amount of the harvested energy is

$$
E_{E H}=\alpha \eta\left(P_{p 1}\|\mathbf{g}\|^{2}+N_{0}\right),
$$

where $\eta$ is the efficiency of EH.

The PU receives

$$
y_{p 1}=h_{p} s_{p 1}+n_{p 1}
$$

where $n_{p 1} \in \mathcal{C N}\left(0, \tilde{N}_{0}\right)$ and achieves a rate of

$$
R_{p 1}=\alpha \log _{2}\left(1+\frac{P_{p 1}\left|h_{p}\right|^{2}}{\tilde{N}_{0}}\right)
$$

In Phase II, the PT sends signals $s_{p 2}\left(\mathrm{E}\left[\left|s_{p 2}\right|^{2}\right]=1\right)$ with average power $P_{p 2}$ to the $\mathrm{ST}$, then the received signals at the $\mathrm{PU}$ and the ST are, respectively,

$$
y_{p 2}=\sqrt{P_{p 2}} h_{p} s_{p 2}+n_{p 2}, \text { and } \mathbf{r}_{2}=\sqrt{P_{p 2}} \mathbf{g} s_{p 2}+\mathbf{n}_{R 2},
$$

where $n_{p 2} \in \mathcal{C} \mathcal{N}\left(0, \tilde{N}_{0}\right)$ and $\mathbf{n}_{R 2} \in \mathcal{C N}\left(\mathbf{0}, \tilde{N}_{0} \mathbf{I}\right)$ are the combined noise received at the PU and the ST, respectively. The ST adopts the same strategy as the power splitting protocol to process the received primary signal, i.e., it applies first an MRC receiver $\mathrm{g}$ then forwards it using a new beamforming vector $\mathbf{w}_{p}$

In Phase III, the ST superimposes the processed primary signal with its own data $s_{s}$ using the cognitive beamforming vector $\mathbf{w}_{s}$, then transmits it to both the PU and the SU. In this phase, the PT remains idle. The ST's transmit signal is written as

$$
\mathbf{t}=\mathbf{w}_{s} s_{s}+\sqrt{P_{p 2}} \mathbf{w}_{p}\|\mathbf{g}\|^{2} s_{p 2}+\mathbf{w}_{p} \mathbf{g}^{\dagger} \mathbf{n}_{R 2},
$$

with average power

$$
p_{R}=\mathrm{E}\left[\|\mathbf{t}\|^{2}\right]=\left\|\mathbf{w}_{s}\right\|^{2}+\left\|\mathbf{w}_{p}\right\|^{2}\left(P_{p 2}\|\mathbf{g}\|^{4}+\|\mathbf{g}\|^{2} \tilde{N}_{0}\right) .
$$

The received signal at the $\mathrm{SU}$ is

$$
\begin{aligned}
y_{s}= & \mathbf{h}_{s}^{\dagger} \mathbf{t}+n_{s}=\mathbf{h}_{s}^{\dagger} \mathbf{w}_{s} s_{s} \\
& +\sqrt{P_{p 2}} \mathbf{h}_{s}^{\dagger} \mathbf{w}_{p}\|\mathbf{g}\|^{2} s_{p 2}+\mathbf{h}_{s}^{\dagger} \mathbf{w}_{p} \mathbf{g}^{\dagger} \mathbf{n}_{R 2}+n_{s},
\end{aligned}
$$

where $n_{s} \in \mathcal{C N}\left(\mathbf{0}, \tilde{N}_{0}\right)$ is the combined noise received at the $\mathrm{SU}$. The received SINR at SU is then expressed as

$$
\Gamma_{s}=\frac{\left|\mathbf{h}_{s}^{\dagger} \mathbf{w}_{s}\right|^{2}}{P_{p 2}\left|\mathbf{h}_{s}^{\dagger} \mathbf{w}_{p}\right|^{2}\|\mathbf{g}\|^{4}+\left|\mathbf{h}_{s}^{\dagger} \mathbf{w}_{p}\right|^{2}\|\mathbf{g}\|^{2} \tilde{N}_{0}+\tilde{N}_{0}},
$$

and the achievable rate is $R_{s}=\frac{1-\alpha}{2} \log _{2}\left(1+\Gamma_{s}\right)$. The received signal at the $\mathrm{PU}$ is

$$
\begin{aligned}
y_{p 3}= & \mathbf{h}_{s p}^{\dagger} \mathbf{t}+n_{p 3}=\mathbf{h}_{s p}^{\dagger} \mathbf{w}_{s} s_{s}+\sqrt{P_{p 2}} \mathbf{h}_{s p}^{\dagger} \mathbf{w}_{p}\|\mathbf{g}\|^{2} s_{p 2} \\
& +\mathbf{h}_{s p}^{\dagger} \mathbf{w}_{p} \mathbf{g}^{\dagger} \mathbf{n}_{R 2}+n_{p 2} .
\end{aligned}
$$

Applying MRC to $y_{p 2}$ and $y_{p 3}$, the received SINR of the $\mathrm{PU}$ is the sum of two channel uses, and considering $R_{p 1}$ in the first phase, the total PU rate is

$$
\begin{aligned}
R_{p}= & \alpha \log _{2}\left(1+\frac{P_{p 1}\left|h_{p}\right|^{2}}{\tilde{N}_{0}}\right)+\frac{1-\alpha}{2} \log _{2}\left(1+\frac{P_{p 2}\left|h_{p}\right|^{2}}{\tilde{N}_{0}}\right. \\
& \left.+\frac{P_{p 2}\left|\mathbf{h}_{s p}^{\dagger} \mathbf{w}_{p}\right|^{2}\|\mathbf{g}\|^{4}}{\left|\mathbf{h}_{s p}^{\dagger} \mathbf{w}_{s}\right|^{2}+\left|\mathbf{h}_{s p}^{\dagger} \mathbf{w}_{p}\right|^{2}\|\mathbf{g}\|^{2} \tilde{N}_{0}+\tilde{N}_{0}}\right) .
\end{aligned}
$$

The problem of maximizing the SU rate with PU rate and ST power constraint is formulated as (47) at the top of next page, where we have imposed the peak power constraint $P_{\max }$ on the PT's transmit power in both Phase I and Phase II, to prevent extremely high transmit power. 


$$
\begin{aligned}
\max _{\alpha, P_{p 1}, \mathbf{w}_{s}, \mathbf{w}_{p}} & \frac{1-\alpha}{2} \log _{2}\left(1+\frac{\left|\mathbf{h}_{s}^{\dagger} \mathbf{w}_{s}\right|^{2}}{P_{p 2}\left|\mathbf{h}_{s}^{\dagger} \mathbf{w}_{p}\right|^{2}\|\mathbf{g}\|^{4}+\left|\mathbf{h}_{s}^{\dagger} \mathbf{w}_{p}\right|^{2}\|\mathbf{g}\|^{2} \tilde{N}_{0}+\tilde{N}_{0}}\right) \\
\text { s.t. } \quad & \alpha \log _{2}\left(1+\frac{P_{p 1}\left|h_{p}\right|^{2}}{\tilde{N}_{0}}\right)+\frac{1-\alpha}{2} \log _{2}\left(1+\frac{P_{p 2}\left|h_{p}\right|^{2}}{\tilde{N}_{0}}+\frac{P_{p 2}\left|\mathbf{h}_{s p}^{\dagger} \mathbf{w}_{p}\right|^{2}\|\mathbf{g}\|^{4}}{\left|\mathbf{h}_{s p}^{\dagger} \mathbf{w}_{s}\right|^{2}+\left|\mathbf{h}_{s p}^{\dagger} \mathbf{w}_{p}\right|^{2}\|\mathbf{g}\|^{2} \tilde{N}_{0}+\tilde{N}_{0}}\right) \geq r_{p}, \\
& P_{p 1} \leq P_{\max }, P_{p 2}=\max \left(P_{\max }, 2 \frac{P_{p}-\alpha P_{p 1}}{1-\alpha}\right), \\
& \left\|\mathbf{w}_{s}\right\|^{2}+\left\|\mathbf{w}_{p}\right\|^{2}\left(P_{p 2}\|\mathbf{g}\|^{4}+\|\mathbf{g}\|^{2} \tilde{N}_{0}\right) \leq 2 \frac{\alpha \eta\left(P_{p 1}\|\mathbf{g}\|^{2}+N_{0}\right)+P_{s 0}}{(1-\alpha)}, \\
& 0 \leq \alpha \leq 1 .
\end{aligned}
$$

Following a similar procedure to obtain (29), problem 47) can be written more compactly as

$$
\begin{aligned}
\underset{\alpha, P_{p_{1}}, \mathbf{w}_{s}, \mathbf{w}_{p}}{\max } & \frac{1-\alpha}{2} \log _{2}\left(1+\frac{\left|\mathbf{h}_{s}^{\dagger} \mathbf{w}_{s}\right|^{2}}{\left|\mathbf{h}_{s}^{\dagger} \mathbf{w}_{p}\right|^{2}+\tilde{N}_{0}}\right) \\
\text { s.t. } \quad & \frac{\left|\mathbf{h}_{s p}^{\dagger} \mathbf{w}_{p}\right|^{2}}{\left|\mathbf{h}_{s p}^{\dagger} \mathbf{w}_{s}\right|^{2}+\tilde{N}_{0}} \geq \frac{\left(P_{p 2}\|\mathbf{g}\|^{2}+\tilde{N}_{0}\right) \gamma_{p}^{\prime}}{\|\mathbf{g}\|^{2}-\gamma_{p}^{\prime} \tilde{N}_{0}}, \\
& P_{p 1} \leq P_{\max }, P_{p 2}=\max \left(P_{\max }, 2 \frac{P_{p}-\alpha P_{p 1}}{1-\alpha}\right), \\
& \left\|\mathbf{w}_{s}\right\|^{2}+\left\|\mathbf{w}_{p}\right\|^{2} \leq 2 \frac{\alpha \eta\left(P_{p 1}\|\mathbf{g}\|^{2}+N_{0}\right)+P_{s 0}}{(1-\alpha)}, \\
& 0 \leq \alpha \leq 1, \\
& 2\left(r_{p}-\alpha \log _{2}\left(1+\frac{P_{p 1}\left|h_{p}\right|^{2}}{N_{0}}\right)\right) \\
\text { where } \gamma_{p}^{\prime} \triangleq & \frac{2}{P_{p 2}}-\frac{\left|h_{p}\right|^{2}}{\tilde{N}_{0}} . \text { Given } \alpha
\end{aligned}
$$
and $P_{p 1}$, the optimal $\mathbf{w}_{s}, \mathbf{w}_{p}$ can be found using Lemma 1. Therefore (48) can be solved by performing 2-D search over $\left(\alpha, P_{p 1}\right)$.

\section{B. ZF Solution}

Similar to the case of power splitting, we study the ZF solution for time splitting which require that $\mathbf{h}_{s}^{\dagger} \mathbf{w}_{p}=\mathbf{h}_{p}^{\dagger} \mathbf{w}_{s}=$ 0 . The simplified problem (48) becomes

$$
\begin{aligned}
\underset{\alpha, P_{p 1}, q_{s}, q_{p}}{\max } & \frac{1-\alpha}{2} \log _{2}\left(1+\frac{q_{s}\left\|\mathbf{h}_{s}\right\|^{2}\left(1-\delta^{2}\right)}{\tilde{N}_{0}}\right) \\
\text { s.t. } & \frac{q_{p}\left\|\mathbf{h}_{s p}\right\|^{2}\left(1-\delta^{2}\right)}{\tilde{N}_{0}} \geq \frac{\left(P_{p 2}\|\mathbf{g}\|^{2}+\tilde{N}_{0}\right) \gamma_{p}^{\prime}}{\|\mathbf{g}\|^{2}-\gamma_{p}^{\prime} \tilde{N}_{0}}, \\
& P_{p 1} \leq P_{\max }, P_{p 2}=\max \left(P_{\max }, 2 \frac{P_{p}-\alpha P_{p 1}}{1-\alpha}\right), \\
& q_{s}+q_{p} \leq 2 \frac{\alpha \eta\left(P_{p 1}\|\mathbf{g}\|^{2}+N_{0}\right)+P_{s 0}}{(1-\alpha)}, \\
& 0 \leq \alpha \leq 1, q_{s} \geq 0, q_{p} \geq 0 .
\end{aligned}
$$

Given $\left(\alpha, P_{p 1}\right)$, the solution to $\left(q_{s}, q_{p}\right)$ is easily derived. However, this leads to a complicated objective function about $\left(\alpha, P_{p 1}\right)$ which does not admit a closed-form solution, therefore the optimal solution can be found by performing 2-D search over $\left(\alpha, P_{p 1}\right)$.

\section{Simulation Results}

In this section the performance results of the proposed primary-secondary cooperation schemes are presented through computer simulation. We assume that the ST has $N=4$ transmit antennas. We consider a scenario where the distances from the ST to all the other terminals are $1 \mathrm{~m}$, while the distance from the PT to the PU is $2 \mathrm{~m}$, therefore assistance from the ST is usually preferred by the PT. The potential application scenarios include wireless sensor networks or the indoor environment where $\mathrm{WiFi}$ and ZigBee coexist both operating at $2.4 \mathrm{GHz}$ which leads to significant interference [31]. WiFi is the primary system and ZigBee is the secondary system. Zigbee wants to share the spectrum occupied by $\mathrm{WiFi}$ but it has very limited energy supply or even no battery. The channel between a transmit-receive antenna pair is modeled as $h=(\Delta)^{-\frac{l}{2}} e^{j \omega}$, where $\Delta$ is the distance, $l$ is the path loss exponent, chosen as 3.5 , and $\omega$ is uniformly distributed over $[0,2 \pi)$. The variance of noise components are normalized to unity, i.e., $N_{0}=N_{C}=1$. The primary energy is set to $P_{p}=20 \mathrm{~dB}$, the peak power is $P_{\max }=30 \mathrm{~dB}$, and the PU rate requirement is $r_{p}=3 \mathrm{bps} / \mathrm{Hz}$, unless otherwise specified. Outage occurs when the required PU rate is not supported. We will evaluate the performance of the proposed schemes including the ideal cooperation (labeled as 'Ideal Cooperation'), the power splitting scheme (labeled as 'Power Splitting EH') and the time splitting scheme with both fixed equal power (labeled as 'Time Splitting EH' ) and adaptive power allocation (labeled as 'Time Splitting EH with Power Allocation') during the energy and information transfer. The case of information cooperation only [14] between the primary and secondary systems without energy cooperation is used as the benchmark (labeled as 'No Energy Cooperation'). Unless otherwise specified, the results are averaged over 1000 channel realizations.

In Fig. 7, we plot the rate regions for different schemes for a specific randomly chosen channel realization $\left|h_{p}\right|^{2}=$ $0.0002, \mathbf{g}=[-0.9472-0.6334 i-0.9090-1.2266 i-$ $1.1855+0.3370 i 0.5345-0.1796 i]^{T}, \mathbf{h}_{s}=[-0.9215-$ $0.4314 i 0.2052-0.2503 i 0.3109-0.3055 i 0.3560+0.1163 i]^{T}$ and $\mathbf{h}_{s p}=[0.3610-0.1248 i 1.1616+0.8211 i-0.4350-$ $0.2818 i-0.4445+0.6564 i]^{T}$. ST's own energy is $P_{s 0}=10$ dB. The efficiency of energy transfer is assumed to be $\eta=0.5$. It is seen that the achievable rate regions are greatly enlarged due to the energy cooperation. It is observed that the power splitting scheme for energy cooperation outperforms the time splitting scheme. Due to the non-causal information transfer, the ideal information and energy cooperation provides 
an outer bound for both practical cooperation schemes.

Next we investigate the impact of the ST's self energy on the achievable average SU rate in Fig. 8 when the efficiency of energy transfer, $\eta$, takes values $0.1,0.5$ and 1 . Substantial rate gain is achieved using the proposed schemes compared with the case without energy cooperation, especially in low to medium ST energy region even when the efficiency is $\eta=0.1$. While in the high energy region and $\eta=0.1$, the performance of the information only cooperation scheme is close to that of the power splitting and time splitting scheme. This is because the ST has sufficient energy and the efficiency of energy transfer is low, there is no need to harvest energy from the primary transmission. It is also observed that the power splitting scheme achieves higher SU rate than the time splitting scheme when the efficiency $\eta=0.5$ and 1 while when $\eta=0.1$, the time splitting scheme outperforms the power splitting scheme in the low to medium energy region.

We then compare the PU rate outage performance of different schemes when the ST energy varies from 0 to $20 \mathrm{~dB}$ in Fig. 9. We assume the $\mathrm{SU}$ rate requirement is $r_{s}=3$ $\mathrm{bps} / \mathrm{Hz}$. It is first noted that without any primary-secondary cooperation, the PU experiences rate outage with a high probability of over $80 \%$ due to the weak primary channel. If only information cooperation but no energy transfer is allowed, the outage probability can be reduced only in the high energy region. When $\eta \geq 0.5$, the power splitting and time splitting schemes achieve outage probabilities of below $20 \%$ and $35 \%$, respectively, which is a substantial improvement.

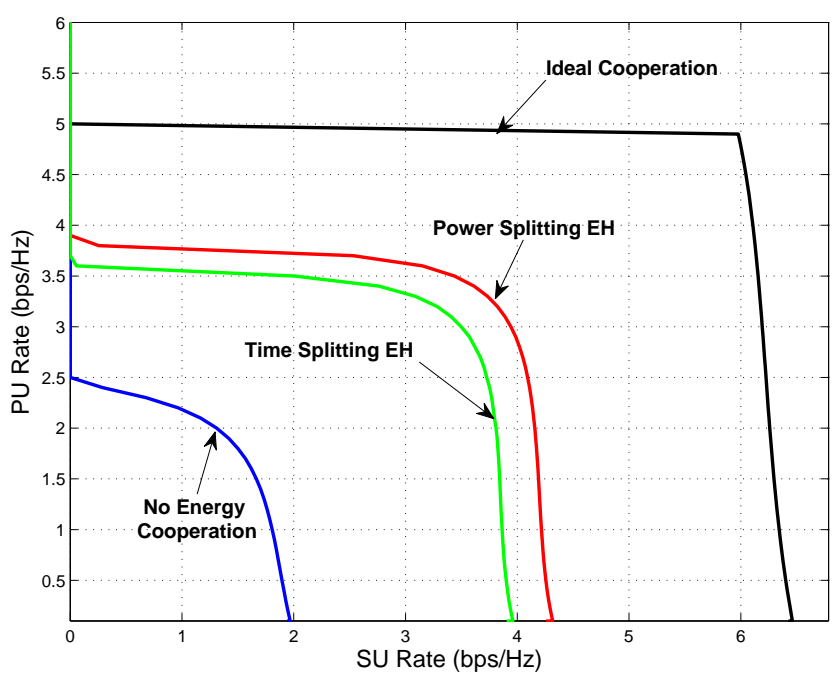

Fig. 7. PU-SU rate region, $P_{p}=20 \mathrm{~dB}, P_{s 0}=10 \mathrm{~dB}$.

\section{CONCLUSIONS}

This paper has investigated energy cooperation between the primary and secondary system in cognitive radio networks, in addition to existing information cooperation. The rationale behind is that the primary system provides spectrum as well energy to the secondary system, and in return the secondary system is more willing and able to assist the primary transmission. This creates more incentives for the primary and

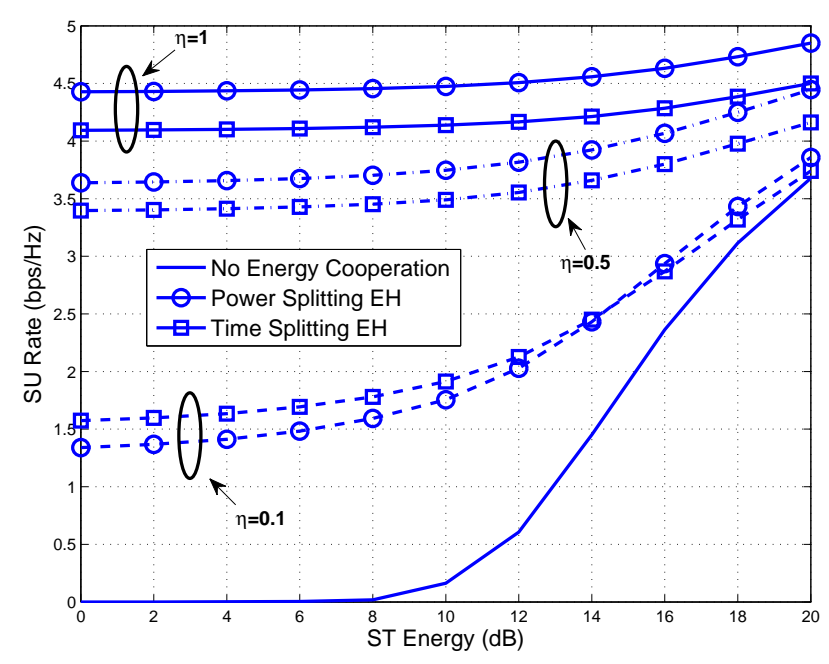

Fig. 8. SU rate vs $\mathrm{SU}$ power, $r_{p}=3 \mathrm{bps} / \mathrm{Hz}$.

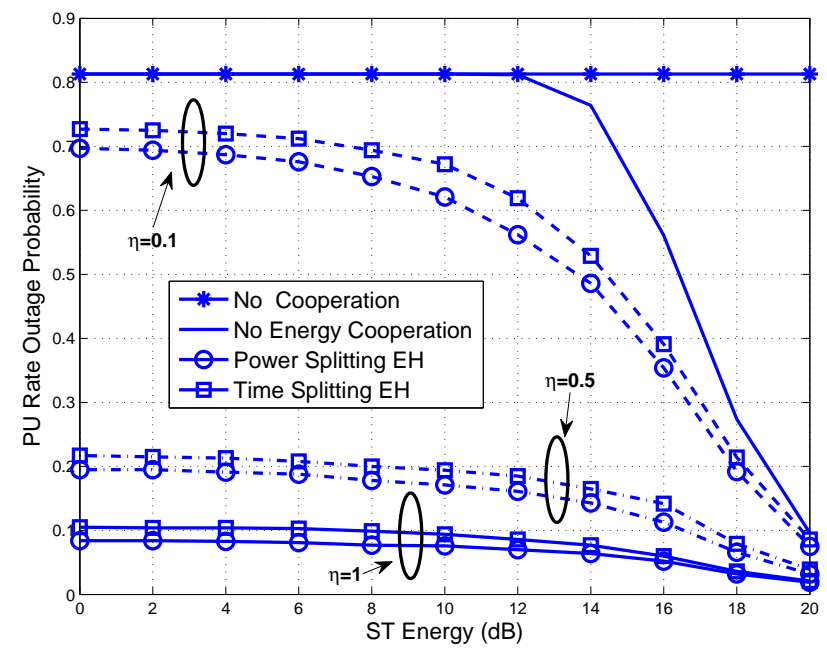

Fig. 9. PU rate outage vs SU power, $r_{p}=3 \mathrm{bps} / \mathrm{Hz}$ and $r_{s}=4 \mathrm{bps} / \mathrm{Hz}$.

secondary systems to cooperate and overall the spectrum is better utilized. We have studied three protocols that enables both energy and information cooperation. The first one is the ideal cooperation assuming non-causal primary information available at the secondary transmitter; the other two protocols employ practical power splitting and time splitting for energy and information transfer. For each scheme, the optimal as well as low-complexity solutions are derived, based on which some insights are drawn on system parameters. It is found that the power splitting scheme usually can support a larger rate region than the time splitting scheme when the efficiency of energy transfer is sufficiently high. Substantial performance gain has been shown using the proposed additional energy cooperation than the existing information cooperation only $\mathrm{CR}$ scheme, therefore energy and information cooperation could be a promising solution for the future CR networks. 


\section{ACKNOWLEDGEMENT}

The authors thank the anonymous reviewers and the associated editor for their useful comments which have substantially improve the quality of the manuscript. The first and the third authors would like to thank David Halls and Aissa Ikhlef from Toshiba Research Europe Limited, for their suggestions to improve the clarity of this manuscript.

\section{APPENDIX A}

\section{PROOF OF PROPOSITION 1}

The maximum PU rate is achieved when SU rate is zero and $\mathbf{w}_{s}=\mathbf{0}$. In this case, $q_{p}=P_{s 0}+\beta \eta P_{p}$ and the optimal $\beta$ that maximizes the PU rate is given by

$\beta^{*}=\arg _{\beta} \max _{0 \leq \beta \leq 1}\left(\sqrt{(1-\beta) P_{p}}\left|h_{p}\right|+\sqrt{P_{s 0}+\beta \eta P_{p}}\left\|\mathbf{h}_{s p}\right\|\right)$.

Setting the derivative to zero leads to the unique critical point

$$
\bar{\beta}=\frac{P_{p} \eta^{2}\left\|\mathbf{h}_{s p}\right\|^{2}-P_{s 0}\left|h_{p}\right|^{2}}{P_{p} \eta^{2}\left\|\mathbf{h}_{s p}\right\|^{2}+\eta P_{p}\left|h_{p}\right|^{2}} .
$$

Then the optimal $\beta$ is $\beta^{*}=[\bar{\beta}]_{0}^{1}$. Accordingly, the maximum PU rate in (6) can be derived.

\section{APPENDIX B}

\section{RECASTING (5) TO THE CONVEX PROGRAM (7)}

We begin by realizing that the optimization problem relates to $\mathbf{w}_{s}$ only in quadratic forms and we can multiply a complex phase to the optimization objective so that the complex phase is offset. Hence, we replace the optimization objective by $\operatorname{Re}\left(\mathbf{h}_{s}^{\dagger} \mathbf{w}_{s}\right)$.

$$
\begin{aligned}
\max _{\mathbf{w}_{s}, \mathbf{w}_{p}, \beta} & \operatorname{Re}\left(\mathbf{h}_{s}^{\dagger} \mathbf{w}_{s}\right) \\
\text { s.t. } & \left|\sqrt{(1-\beta) P_{p}}\right| h_{p}\left|+\mathbf{h}_{s p}^{\dagger} \mathbf{w}_{p}\right|^{2} \\
& \geq \gamma_{p}\left(\left|\mathbf{h}_{s p}^{\dagger} \mathbf{w}_{s}\right|^{2}+\tilde{N}_{0}\right), \\
& \left\|\mathbf{w}_{s}\right\|^{2}+\left\|\mathbf{w}_{p}\right\|^{2} \leq P_{s 0}+\beta \eta P_{p},
\end{aligned}
$$

where $\gamma_{p}=2^{R_{p}}-1$ is the target SINR. The optimal direction of $\mathbf{w}_{p}=\sqrt{q_{p}} \frac{\mathbf{h}_{s p}}{\left\|\mathbf{h}_{s p}\right\|} e^{j \arg \left(h_{p}\right)}$ can be determined and what is left for optimizing is the power of $\mathbf{w}_{p}, q_{p}$. Thus we have

$$
\begin{aligned}
\max _{\mathbf{w}_{s}, q_{p}, \beta} & \operatorname{Re}\left(\mathbf{h}_{s}^{\dagger} \mathbf{w}_{s}\right) \\
\text { s.t. } & \left(\sqrt{(1-\beta) P_{p}}\left|h_{p}\right|+\sqrt{q_{p}}\left\|\mathbf{h}_{s p}\right\|\right)^{2} \\
& \geq \gamma_{p}\left(\left|\mathbf{h}_{s p}^{\dagger} \mathbf{w}_{s}\right|^{2}+\tilde{N}_{0}\right), \\
& \left\|\mathbf{w}_{s}\right\|^{2}+q_{p} \leq P_{s 0}+\beta \eta P_{p} .
\end{aligned}
$$

Now, we let $\bar{\beta}=\sqrt{1-\beta}$ and $\bar{q}_{p}=\sqrt{q_{p}}$ and the ranges of the parameters remain the same: $0 \leq \bar{\beta} \leq 1$ and $\overline{q_{p}} \geq 0$. The optimization problem becomes

$$
\begin{aligned}
\max _{\mathbf{w}_{s}, \bar{q}_{p}, \bar{\beta}} & \operatorname{Re}\left(\mathbf{h}_{s}^{\dagger} \mathbf{w}_{s}\right) \\
\text { s.t. } & \left(\bar{\beta} \sqrt{P_{p}}\left|h_{p}\right|+\bar{q}_{p}\left\|\mathbf{h}_{s p}\right\|\right)^{2} \geq \gamma_{p}\left(\left|\mathbf{h}_{s p}^{\dagger} \mathbf{w}_{s}\right|^{2}+\tilde{N}_{0}\right), \\
& \left\|\mathbf{w}_{s}\right\|^{2}+\bar{q}_{p}^{2} \leq P_{s 0}+\left(1-\bar{\beta}^{2}\right) \eta P_{p}, \\
& 0 \leq \bar{\beta} \leq 1, \overline{q_{p}} \geq 0 .
\end{aligned}
$$

Define a vector $\mathbf{v}$ :

$$
\mathbf{v}=\left[\bar{\beta}, \bar{q}_{p}\right]^{\mathrm{T}}
$$

and the result follows.

\section{APPENDIX C \\ PROOF OF PROPOSITION 2}

Define $q_{s} \triangleq\left\|\mathbf{w}_{s}\right\|^{2}$. First notice that the PU rate and ST power constraints in (5) should be satisfied with equalities. So we have

$$
q_{s}=\left(P_{s 0}+\beta \eta P_{p}\right)-q_{p} .
$$

According to Proposition 1 in [8], the optimal $\mathbf{w}_{s}$ can be parametrized as

$\mathbf{w}_{s}=\sqrt{q_{s}}\left(\sqrt{\lambda} \frac{\Pi_{\mathbf{h}_{s p}} \mathbf{h}_{s}}{\left\|\Pi_{\mathbf{h}_{s p}} \mathbf{h}_{s}\right\|}+\sqrt{1-\lambda} \frac{\Pi_{\mathbf{h}_{s p}}^{\perp} \mathbf{h}_{s}}{\left\|\Pi_{\mathbf{h}_{s p}}^{\perp} \mathbf{h}_{s}\right\|}\right), 0 \leq \lambda \leq 1$.

We then have $\left|\mathbf{h}_{s p}^{\dagger} \mathbf{w}_{s}\right|^{2}=\lambda q_{s}\left\|\mathbf{h}_{s p}\right\|^{2}$. Substituting it into the PU rate constraint in (5), we can solve $\lambda$ as:

$$
\lambda=\frac{\frac{\left|\sqrt{(1-\beta) P_{p}}\right| h_{p}\left|+\sqrt{q_{p}}\left\|\mathbf{h}_{s p}\right\|\right|^{2}}{2^{r} p-1}-\tilde{N}_{0}}{\left(\left(P_{s 0}+\beta \eta P_{p}\right)-q_{p}\right)\left\|\mathbf{h}_{s p}\right\|^{2}} .
$$

Substituting (54) - (56) into the objective function in (5) gives the formulation (8).

\section{APPENDIX D}

\section{FEASIBILITY RANGE IN POWER-SPLITTING COOPERATION}

Define $a_{1} \triangleq 2 P_{p}\|\mathbf{g}\|^{2}+N_{0}, \quad b_{1} \triangleq 2 P_{s 0}, \quad c_{1} \triangleq\left\|\mathbf{h}_{s p}\right\|^{2}$, then (28) is equivalent to

$$
\begin{aligned}
\min _{\rho} & f_{1}(\rho) \triangleq \frac{\tilde{N}_{0}\left(a_{1}+\frac{N_{C}}{\rho}\right)}{c_{1}\left[b_{1}+a_{1} \eta(1-\rho)\right]}+\left(N_{0}+\frac{N_{C}}{\rho}\right)(57) \\
\text { s.t. } & 0 \leq \rho \leq 1 .
\end{aligned}
$$

The first-order derivative of $f_{1}(\rho)$ is given by

$$
\begin{aligned}
& \frac{\partial f_{1}(\rho)}{\partial \rho} \\
= & -\frac{\tilde{N}_{0} N_{C}}{c_{1}\left[b_{1}+a_{1} \eta(1-\rho)\right] \rho^{2}}+\frac{\tilde{N}_{0}\left(a_{1} \rho+N_{C}\right) a_{1} \eta}{c_{1}\left[b_{1}+a_{1} \eta(1-\rho)\right]^{2} \rho}-\frac{N_{C}}{\rho^{2}},
\end{aligned}
$$

and setting it to zero leads to

$$
f_{2}(\rho) \triangleq A_{1} \rho^{2}+B_{1} \rho-C_{1}=0
$$

where for convenience, we have defined $A_{1} \triangleq a_{1}^{2} \eta \frac{\tilde{N}_{0}}{N_{C}}-$ $\left(a_{1} \eta\right)^{2} c_{1}, B_{1} \triangleq 2 a_{1} \eta N_{0}+2\left(b_{1}+a_{1} \eta\right) a_{1} \eta c_{1}, C_{1} \triangleq N_{0}\left(b_{1}+\right.$ $\left.a_{1} \eta\right)+c_{1}\left(b_{1}+a_{1} \eta\right)^{2}$,

Observe that $f_{2}(\rho)$ has the same sign as $\frac{\partial f(\rho)}{\partial \rho}$ in order to find the optimal $\rho^{*}$. Next we discuss the roots of $f_{2}(\rho)$. Because

$$
\begin{aligned}
& B_{1}^{2}+4 A_{1} C_{1}=\left(2 a_{1} \eta N_{0}+2\left(b_{1}+a_{1} \eta\right) a_{1} \eta c_{1}\right)^{2} \\
& +4\left(a_{1}^{2} \eta \frac{\tilde{N}_{0}}{N_{C}}-\left(a_{1} \eta\right)^{2} c_{1}\right)\left(N_{0}\left(b_{1}+a_{1} \eta\right)+c_{1}\left(b_{1}+a_{1} \eta\right)^{2}\right) \\
> & \left(2 a_{1} \eta N_{0}+2\left(b_{1}+a_{1} \eta\right) a_{1} \eta c_{1}\right)^{2} \\
& -4\left(a_{1} \eta\right)^{2} c_{1}\left(N_{0}\left(b_{1}+a_{1} \eta\right)+c_{1}\left(b_{1}+a_{1} \eta\right)^{2}\right) \\
= & 4 a_{1}^{2} \eta^{2}\left(N_{0}^{2}+c_{1} N_{0}\left(b_{1}+a_{1} \eta\right)\right)>0
\end{aligned}
$$


there are always two distinct real roots $\rho_{1}=\frac{-B_{1}+\sqrt{B_{1}^{2}+4 A C}}{2 A_{1}}$ and $\rho_{2}=\frac{-B_{1}-\sqrt{B_{1}^{2}+4 A C}}{2 A_{1}}$. Depending on the sign of $A_{1}$, there are three possible cases for the optimal $\rho$ :

i) $A_{1}>0$ or $\frac{\tilde{N}_{0}}{N_{C}}>\eta\left\|\mathbf{h}_{s p}\right\|^{2}$. Because $C_{1}>0, \rho_{1}>$ $0, \rho_{2}<0$, therefore $\rho^{*}=\min \left(\rho_{1}, 1\right)$.

ii) $A_{1}<0$ or $\frac{\tilde{N}_{0}}{N_{C}}<\eta\left\|\mathbf{h}_{s p}\right\|^{2}$. In this case, $\rho_{2}>\rho_{1}>0$. Due to the fact that $A_{1}+C_{1}>0$ or $\frac{-C_{1}}{A_{1}}=\rho_{1} \rho_{2}>1$, we know that $\rho_{2}>1$ and it cannot be the optimal solution. Therefore, $\rho^{*}=\min \left(\rho_{1}, 1\right)$.

iii) $A_{1}=0$. In this case, $\rho^{*}=\min \left(\frac{C_{1}}{B_{1}}, 1\right)=$ $\min \left(\frac{P_{s 0}}{2\left(2 P_{p}\|\mathbf{g}\|^{2}+N_{0}\right) \eta}+\frac{1}{2}, 1\right)$.

After finding $\rho^{*}$, the maximum achievable PU rate can be calculated and compared with the PU rate requirement to check the feasibility.

\section{APPENDIX E \\ FEASIBILITY RANGE OF $\rho$ IN POWER SPLITTING COOPERATION}

A given $\rho$ can result in a feasible solution only if the ST can satisfy the PU's rate requirement even without serving the SU. In this extreme case, $\mathbf{w}_{s}=0$ and $\mathbf{w}_{p}=\frac{\sqrt{2 P_{s 0}+P_{E H}} \mathbf{h}_{s p}}{\left\|\mathbf{h}_{s p}\right\|}$. The PU's rate constraint then amounts to

$$
\begin{aligned}
\left(2 P_{s 0}\right. & \left.+\eta(1-\rho)\left(2 P_{p}\|\mathbf{g}\|^{2}+N_{0}\right)\right)\left\|\mathbf{h}_{s p}\right\|^{2} \\
& \geq \frac{\left(2 P_{p} \rho\|\mathbf{g}\|^{2}+\rho N_{0}+N_{C}\right) \gamma_{p}^{\prime} \tilde{N}_{0}}{\rho\|\mathbf{g}\|^{2}-\gamma_{p}^{\prime}\left(\rho N_{0}+N_{C}\right)} .
\end{aligned}
$$

For convenience, define $a \triangleq \eta\left(2 P_{p}\|\mathbf{g}\|^{2}+N_{0}\right), b \triangleq\|\mathbf{g}\|^{2}-$ $\gamma_{p}^{\prime} N_{0}, c \triangleq \frac{\gamma_{p}^{\prime} \tilde{N}_{0}}{\left\|\mathbf{h}_{s p}\right\|^{2}}$ and $A \triangleq a b, B \triangleq-a \gamma_{p}^{\prime} N_{C}-b\left(2 P_{s 0}+\right.$ $a)+a c, C \triangleq N_{C}\left(\gamma_{p}^{\prime}\left(2 P_{s 0}+a\right)+c\right)$. Rearranging the above inequality 610 leads to

$$
2 P_{s 0}+a-a \rho-c \frac{\frac{a \rho}{\eta}+N_{C}}{b \rho-\gamma_{p}^{\prime} N_{C}} \geq 0,
$$

and

$$
f_{P S}(\rho) \triangleq A \rho^{2}+B \rho+C \leq 0 .
$$

We then discuss possible cases below.

- $A=0$ or $\|\mathbf{g}\|^{2}=N_{0}\left(\frac{2^{2 r_{p}}-1}{2 P_{p}}-\frac{\left|h_{p}\right|^{2}}{N_{0}}\right)$. Under this condition, there are two possibilities:

- $c \geq \gamma_{p}^{\prime} \eta N_{C}$ or $\tilde{N}_{0} \geq \eta N_{C}\left\|\mathbf{h}_{s p}\right\|^{2}$. In this case, $0 \leq$ $\rho \leq 1$.

- $c<\gamma_{p}^{\prime} \eta N_{C}$ or $\tilde{N}_{0}<\eta N_{C}\left\|\mathbf{h}_{s p}\right\|^{2}$, then $0 \leq \rho<$ $\left[\frac{2 P_{s 0}+a+\tilde{N}_{0}\left\|\mathbf{h}_{s p}\right\|^{2}}{a-\frac{N_{0}\left\|\mathbf{h}_{s p}\right\|^{2} a}{\eta N_{C}}}\right]_{0}^{1}$.

When $A \neq 0$, there are two possible cases:

- If the discriminant is nonnegative, suppose the two real roots of the quadratic equation $f_{P S}=0$ are given by $\bar{\rho}_{\text {min }}$ and $\bar{\rho}_{\text {max }}$, and $\bar{\rho}_{\text {min }} \leq \bar{\rho}_{\text {max }}$. We have the following discussion.

- $A>0$. The feasible $\rho$ should satisfy $\left[\bar{\rho}_{\text {min }}\right]_{0}^{1} \leq \rho \leq$ $\left[\bar{\rho}_{\max }\right]_{0}^{1}$.

- $A<0$. Because $C>0$, we know that $\bar{\rho}_{\text {min }}<0$ and $\bar{\rho}_{\max }>0$, thus we have $\left[\bar{\rho}_{\max }\right]_{0}^{1} \leq \rho \leq 1$.

- If the discriminant is negative, there is no feasible $\rho$.

\section{APPENDIX F}

OPTIMALITY OF $\rho_{z f}^{*}$ IN POWER SPLITTING COOPERATION

Because the first two constraints in (34) should hold with equality, we can derive

$$
\begin{aligned}
q_{s}(\rho) & =2 P_{s 0}+a-a \rho-c_{z f} \frac{a \rho+N_{C}}{b \rho-\gamma_{p}^{\prime} N_{C}} \\
& =2 P_{s 0}+a-a \rho-\frac{a c}{b}-\frac{\left(\gamma_{p}^{\prime} \frac{a}{b}+1\right) N_{C} c_{z f}}{b \rho-\gamma_{p}^{\prime} N_{C}}
\end{aligned}
$$

where $c_{z f}=\frac{\gamma_{p}^{\prime} \tilde{N}_{0}}{\left\|\mathbf{h}_{s p}\right\|^{2}\left(1-\delta^{2}\right)}$. Maximizing $q_{s}$ is equivalent to

$$
\min _{0<\rho<1} a \rho+\frac{\left(\gamma_{p}^{\prime} \frac{a}{b}+1\right) N_{C} c_{z f}}{b \rho-\gamma_{p}^{\prime} N_{C}}
$$

whose minimum is achieved by (35). To compare with the optimal solution, we also study the feasible range of $\rho$ for the ZF scheme. A given $\rho$ is feasible requires that $q_{s}(\rho) \geq 0$ or

$$
f_{P S-Z F}(\rho) \triangleq A \rho^{2}+B \rho+C_{z f} \leq 0,
$$

where $C_{z f} \triangleq N_{C}\left(\gamma_{p}^{\prime}\left(2 P_{s 0}+a\right)+c_{z f}\right)$. Similar results as the optimal case (66) can be obtained, except replacing $C$ with $C_{z f}$. Since $C_{z f}>C$ due to the fact that $c_{z f}>c$, the optimal scheme has a larger feasibility region for $\rho$ than the ZF solution.

\section{REFERENCES}

[1] J. Mitola, "Cognitive Radio: An Integrated Agent Architecture for Software Defined Radio," PhD Dissertation, KTH, Stockholm, Sweden, Dec. 2000.

[2] S. Haykin, "Cognitive Radio: Brain-Empowered Wireless Communications," IEEE J. Sel. Areas Commun., vol. 23, pp. 201-220, Feb. 2005.

[3] A. K. Sadek, K.J.R. Liu, and A. Ephremides, "Cognitive multiple access via cooperation: Protocol design and stability analysis," IEEE Trans. Inf. Theory, vol. 53, no. 10, pp. 3677-3696, Oct. 2007.

[4] N. Devroye, P. Mitran, and V. Tarokh, "Achievable rates in cogtive radio channels," IEEE Trans. Inf. Theory, vol. 52, no. 5, pp. 1813-1827, May 2006.

[5] S. Srinivasa and S. A. Jafar, "The throughput potential of cognitive radio-a theoretical perspective," IEEE Commun. Mag., vol. 45, no. 5, pp. 73-79, May 2007.

[6] A. Jovicic and P. Viswanath, "Cognitive radio: An information-theoretic perspective," IEEE Trans. Inf. Theory, vol. 55, no. 9, pp. 3945-3958, Sept. 2009.

[7] J. Lv, R. Blasco-Serrano, E. A. Jorswieck, R. Thobaben and A. Kliks, "Optimal Beamforming in MISO Cogntive Channels with Degraded Message Sets," in Proc. IEEE Wireless Commun. Netw. Conf. (WCNC), Paris, France, 1-4 Apr. 2012, pp. 538-543 .

[8] J. Lv and E. A. Jorswieck, "Spatial shaping in cognitive system with coded legacy transmission," in Proc. Int. ITG Workshop on Smart Antennas (WSA), Aachen, Germany, 24-25 Feb. 2011, pp. 1-6.

[9] R. Blasco-Serrano, J. Lv, R. Thobaben, E. A. Jorswieck and M. Skoglund, "Multi-antenna Transmission for Underlay and Overlay Cognitive Radio with Explicit Message Learning Phase," EURASIP Journal on Wireless Communications and Networking (JWCN), special issue on "Cooperative Cognitive Networks", 2013:195, doi:10.1186/1687-14992013-195.

[10] O. Simeone, I. Stanojev, S. Savazzi, Y. Bar-Ness, U. Spagnolini, and R. Pickholtz, "Spectrum leasing to cooperating secondary ad hoc networks," IEEE J. Sel. Areas Commun., vol. 26, no. 1, pp. 203 -213, Jan. 2008.

[11] W. Su, J. D. Matyjas, and S. N. Batalama, "Active cooperation between primary users and cognitive radio users in heterogeneous ad-hoc networks," IEEE Trans. Signal Process., vol. 60, no. 4, pp.1796-1805, Apr. 2012.

[12] R. Manna, Raymond H. Y. Louie. Y. Li, and B. Vucetic, "Cooperative Spectrum Sharing in Cognitive Radio Networks With Multiple Antennas," IEEE Trans. Signal Process., vol. 59, no. 11, pp. 5509-5522, Nov. 2011. 
[13] S.H. Song, M. O. Hasna, and K. B. Letaief, "Prior zero-forcing for cognitive relaying," IEEE Trans. Wireless Commun., vol. 12, no. 2, pp. 938 - 947, Feb. 2013.

[14] G. Zheng, S.H. Song, K. K. Wong, and B. Ottersten, "Cooperative Cognitive Networks: Optimal, Distributed and Low-Complexity Algorithms", IEEE Trans. Sig. Process., vol. 61, no. 11, pp. 2778 - 2790, June 2013.

[15] B. Cao, L. X. Cai, L. Hao, J. W. Mark, Q. Zhang, H.V. Poor, and W. Zhuang , "Cooperative Cognitive Radio Networking Using Quadrature Signaling," in Proc. INFOCOM, Orlando, FL., March. 2012, pp. 30963100 .

[16] G. Zheng, I. Krikidis, and B. Ottersten, "Full-Duplex Cooperative Cognitive Radio with Transmit Imperfections," IEEE Trans. Wireless Commun., vol. 12, no. 5, pp. 2498 - 2511, May 2013.

[17] R. Zhang and C. K. Ho, "MIMO broadcasting for simultaneous wireless information and power transfer," IEEE Trans. Wireless Communs., vol. 12, no. 5, pp. 1989-2001, May, 2013.

[18] X. Zhou, R. Zhang, and C. K. Ho, "Wireless information and power transfer: architecture design and rate-energy tradeoff," submitted to IEEE Trans. Wireless Communs., available at http://arxiv.org/abs/1205.0618

[19] J. Xu, Y. H. Guo, and R. Zhang, "CoMP meets energy harvesting: a new communication and energy cooperation paradigm," submitted to IEEE Global Communications Conference (Globecom), 2013, available at http://arXiv/abs/1303.2870

[20] Y. K. Chia, S. Sun, and R. Zhang, "Energy cooperation in cellular networks with renewable powered base stations," in IEEE Wireless Communications and Networking Conference (WCNC), Shanghai, China, April 2013.

[21] POWERCAST Frequently Asked Questions. http://www.powercastco.com/frequently-asked-questions/

[22] A. Parks, A. Sample, Y. Zhao, and J. R. Smith, "A wireless sensing platform utilizing ambient rf energy," in IEEE Topical Meeting on Wireless Sensors and Sensor Networks (WiSNET), Austin, TX, Jan 2013, pp. 1-3.

[23] L. R. Varshney, "Transporting Information and Energy Simultaneously," in Proc. IEEE Int. Symp. Inf. Theory, Toronto, Canada, 6-11 July 2008, pp. 1612-1616.

[24] P. Grover and A. Sahai, "Shannon meets Tesla: wireless information and power transfer," in Proc. IEEE Int. Symp. Inf. Theory, Austin, Texas, USA, June 2010, pp. 2363-2367.

[25] R. Zhang and C. K. Ho, "MIMO broadcasting for simultaneous wireless information and power transfer," in Proc. IEEE Global Commun. Conf. (Globecom), Houston, USA, Dec. 2011, pp. 1-5.

[26] L. Liu, R. Zhang, and K.-C. Chua, "Wireless information transfer with opportunistic energy harvesting," IEEE Trans. Wireless Commun., vol. 12, no. 1, pp. 288-300, Jan. 2013.

[27] I. Krikidis, S. Timotheou, and S. Sasaki, "RF energy transfer for cooperative networks: data relaying or energy harvesting?" IEEE Commun. Lett., vol. 16, no. 11, pp. 1772 - 1775, Nov. 2012.

[28] B. Gurakan, O. Ozel, J. Yang, and S. Ulukus, "Energy Cooperation in Energy Harvesting Communications," IEEE Trans. Commun., to appear. Available at http://www.ece.umd.edu/ ulukus/papers/journal/energy-coop.pdf

[29] A. A. Nasir, X. Zhou, S. Durrani, and R. A. Kennedy, "Relaying Protocols for Wireless Energy Harvesting and Information Processing", IEEE Trans. Wireless Commun., vol. 12, no. 7, pp. 3622 - 3636, July 2013.

[30] M. Schubert and H. Boche, "A generic approach to QoS-based transceiver optimization," IEEE Trans. Commun., vol. 55, no. 8, pp. 1557-1566, Aug. 2007.

[31] J. Huang, G. Xing, G. Zhou and R. Zhou, "Beyond Co-existence: Explo iting WiFi White Space for ZigBee Performance Assurance, " in Proc. IEEE Int. Conf. Network Protocols (ICNP), Kyoto, Japan. Oct. 2010, pp. 305-314. 